\section{Pathologies osseuses des maxillaires récidivantes}

Attitude pratique

Authors:

Olszewski R (DDS, MD, PhD, Prof) ${ }^{1, *}$,

Reychler H (MD, DMD, Dhc, Prof) ${ }^{1}$

\title{
Institutions:
}

${ }^{1}$ Service de stomatologie et de chirurgie maxillo-faciale, Cliniques universitaires saint Luc, Université catholique de Louvain, Bruxelles, Belgique

Auteur correspondant: Olszewski R, Service de stomatologie et de chirurgie maxillo-faciale, Cliniques universitaires saint Luc, Université catholique de Louvain, Av. Hippocrate 10, 1200 Bruxelles, Belgique,

raphael.olszewski@uclouvain.be; téléphone: +3227645718; numéro de fax: +3227645876; ORCID iD: orcid.org/0000-0002-2211-7731

Notice: les opinions exprimées dans ce manuscrit sont propres aux auteurs et il ne s'agit pas d'une opinion officielle d'une institution ou d'une source de financement. 
20

\begin{abstract}
Monsieur,
Veillez accepter pour évaluation ouverte par les pairs, notre article intitulé: «Pathologies osseuses des maxillaires récidivantes: attitude pratique ».

1) Résumé de la contribution de cet article à la littérature scientifique: nous proposons la plus vaste revue de la littérature sur le sujet des pathologies osseuses des maxillaires récidivantes, et nous y adjoignons également la description radiographique et proposons une attitude pratique face à cette problématique.

2) Expliquer le lien entre cette étude et les travaux déjà publiés: les revues de la littérature existants sont concentrés sur une seule pathologie sans donner un diagnostic différentiel et un aperçu global de ce type de pathologie.

3) Spécifier le type d'article (par exemple: article original-de recherché, revue, méta-analyse, essai clinique) : il s'agit d'une revue de la littérature des pathologies les plus fréquentes récidivantes des maxillaires.

4) Décrire des contacts avec la revue Nemesis concernant le manuscript soumis: il s'agit de la prèmiere soumission d'article dans la revue Nemesis.

5) Préciser en quoi l'article correspond aux attentes et objectifs de la revue Nemesis: la revue Nemesis accepte les articles et revues sur les pathologies récidivantes, ce qui est le cas de cet article.
\end{abstract}




\section{Résumé}

Plusieurs pathologies osseuses des maxillaires ont comme caractéristique une tendance à la récidive, et ce même après un traitement approprié. Il convient donc de les reconnaître afin de pouvoir les surveiller, à la fois cliniquement et par une technique d'imagerie appropriée. On distinguera ici des pathologies tumorales bénignes et malignes, métaboliques, malformatives et infectieuses. Actuellement, le CT scan est la technique d'imagerie médicale de premier choix pour établir le diagnostic différentiel et permettre la surveillance de ces pathologies osseuses. Le traitement de ces pathologies récidivantes est soit le curetage/énucléation si possible (corticales non rompues) soit une résection interruptrice ou non des maxillaires ou de la mandibule, et ce en fonction des données cliniques et surtout anatomopathologiques à discuter et envisager au cas par cas, la généralisation dans ce domaine étant impossible voire dangereuse pour le patient. Il ne peut donc être question de proposer des recommandations ni des algorithmes décisionnels. Mots clés: pathologies osseuses, récidive, maxillaire, mandibule

\section{Summary}

Maxillofacial bone diseases represent a heterogeneous group involving benign and malign tumors, and metabolic, malformative, and infectious diseases. They have a tendency to relapse even with a good initial treatment. Therefore, we need to start with active surveillance based on medical imaging (CT scanner). The treatment of maxillofacial bone diseases is based mainly on clinical examination, radiological findings, and pathological diagnosis. The treatment consists of curettage or maxillary/mandibulary interruptive or not-interruptive bone resection. Development of guidelines seems impossible due to the heterogenicity of clinical presentations. The individualized treatment should prevail.

Keywords: bone disease, relapse, review, maxilla, mandible 


\section{Introduction}

Plusieurs pathologies osseuses des maxillaires ont comme caractéristique une tendance à la récidive, et ce même après un traitement approprié. Il convient donc de les reconnaître afin de pouvoir les surveiller, à la fois cliniquement et par une technique d'imagerie appropriée. On distinguera ici des pathologies tumorales bénignes et malignes, métaboliques, malformatives et infectieuses. Actuellement, la tomographie computérisée (CT scan) est la technique d'imagerie médicale de premier choix pour établir le diagnostic différentiel de ces pathologies osseuses, l'orthopantomogramme ne fournissant la plupart du temps que des éléments de suspicion de bénignité ou de malignité, d'origine odontogène ou non (Figure 1). Dans certains cas, l'imagerie par résonance magnétique nucléaire (IRM) permet d'apporter quelques éléments supplémentaires lors d'un diagnostic différentiel insuffisant par l'apport du CT scan, des caractéristiques d'IRM (Figure 2) étant plus et mieux corrélées au contenu des tumeurs que le CT scan. Dans la plupart de ces pathologies, la surveillance radiologique se fait annuellement par un cliché orthopantomographique, et dès qu'une suspicion de récidive apparaît, le bilan sera effectué par un CT scan.

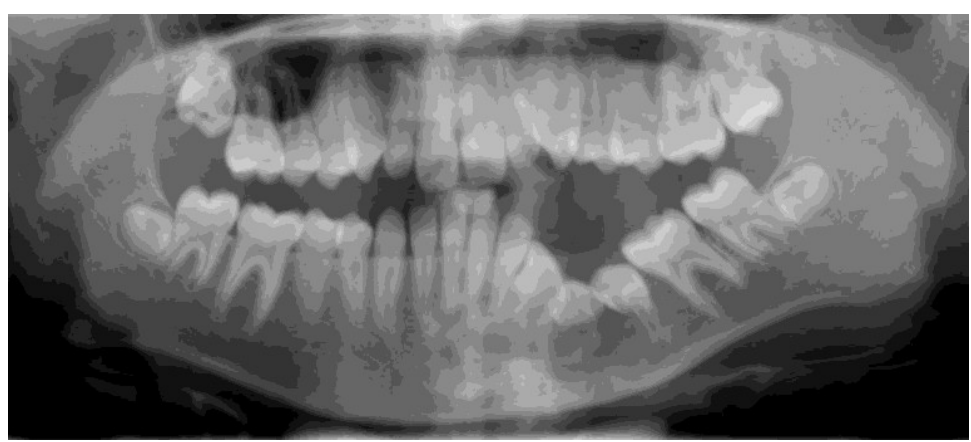

Fig. 1. Orthopantomogramme illustrant une tumeur de la branche horizontale gauche de la mandibule, ayant des répercussions sur l'éruption dentaire et sur la résorption radiculaire de certaines dents. Seule une histologie (difficile la plupart du temps) fournira un diagnostic précis. 


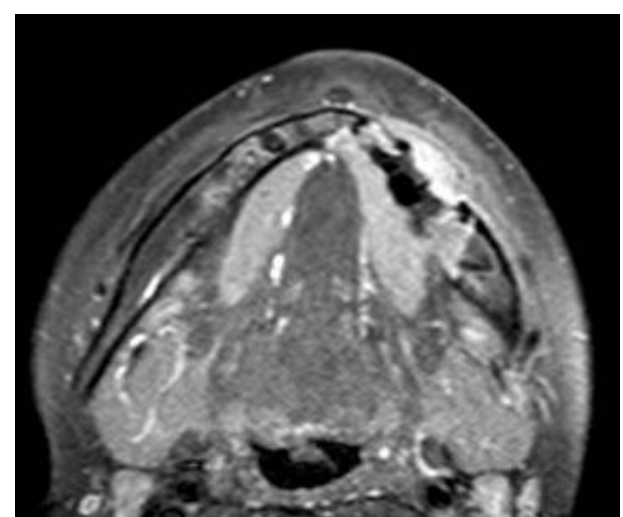

Fig. 2. IRM du même cas illustré ci-dessus.

Grâce aux caractéristiques particulières en IRM, le radiologue peut donner des informations complémentaires très utiles voire pathognomoniques dans certains cas. Le traitement de ces pathologies récidivantes est soit le curetage/énucléation (Figure 3) si possible (corticales non rompues) soit une résection interruptrice ou non des maxillaires ou de la mandibule, et ce en fonction des données cliniques et surtout anatomopathologiques à discuter et envisager au cas par cas, la généralisation dans ce domaine étant impossible voire dangereuse pour le patient. Il ne peut donc être question de proposer des recommandations ni des algorithmes décisionnels.

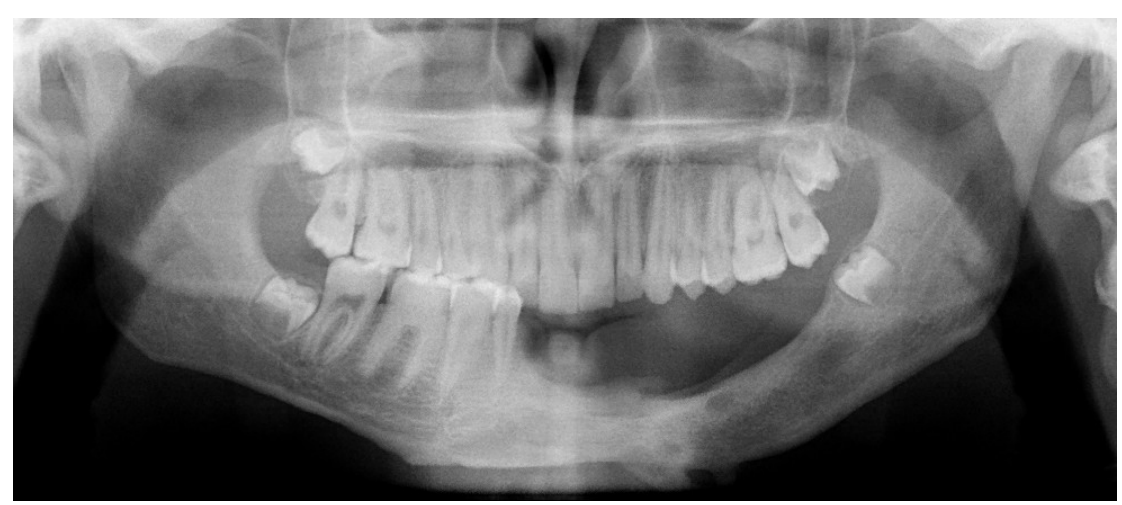

Fig. 3. Orthopantomogramme de la même tumeur myxofibromateuse que celle illustrée en Figure 1, après curetage appuyé effectué à deux reprises en raison d'une première récidive. Le curetage même répété semble souvent moins lourd pour le patient qu'une résection d'emblée interruptrice de la mandibule. 


\section{Kératokystes odontogènes}

Ce sont des kystes avec des caractéristiques histologiques bien définies, ils représentent environ $5 \%$ de toutes les lésions kystiques des maxillaires. Les kératokystes ont leur origine dans la lame dentaire, quand elle possède encore un potentiel de croissance, ou dans la prolifération d'un reste de l'épithélium buccal, sans que le stimulus déclenchant ne soit connu. Ils sont très rares avant l'âge de 10 ans; le pic d'incidence se situe entre les deuxième et troisième décennies. Les hommes en sont atteints deux fois plus que les femmes [1]. La localisation est nettement plus souvent mandibulaire ( $75 \%$ des cas) que maxillaire (Figure 4$)$. A la mandibule, la localisation dans la branche montante et dans la région de la troisième molaire est la plus fréquente, suivie des localisations dans la région des première et deuxième molaires et des localisations plus antérieures; aux maxillaires supérieurs, c'est surtout la région de la troisième molaire puis la région canine qui sont affectées. Des kératokystes multiples sont trouvés en association avec d'autres malformations, dans le syndrome de Gorlin-Goltz (Figure 5); sa transmission est autosomique dominante, avec une pénétrance élevée et une expressivité variable [2]. Il est constitué par une nævomatose basocellulaire qui subit des transformations carcinomateuses et d'autres anomalies cutanées, par des kératokystes des maxillaires, par des anomalies du squelette, en particulier des vertèbres et des côtes, et par des calcifications cérébrales. La face est caractérisée par des bosses frontales ou fronto-pariétales et une base de nez large et aplatie. Cliniquement, le kératokyste se manifeste par une voussure osseuse progressive, qui peut être douloureuse. Les phénomènes inflammatoires ne sont pas rares, avec fistulisation éventuelle. Radiologiquement, la lésion apparaît comme une clarté uni- ou multiloculaire bien délimitée, définie en bordure par un liséré plus dense d'os réactionnel. Une dent incluse peut se trouver dans le voisinage du kyste. Le kyste déplace plus les dents voisines qu'il ne résorbe leurs racines. Les kystes volumineux soufflent l'os et amincissent les corticales. Les kératokystes englobent fréquemment une dent incluse. L'électrophorèse du liquide d'aspiration montre une quantité de protéines solubles inférieure à $4 \mathrm{~g} / 100 \mathrm{ml}$, ce qui incite certains auteurs, à recourir à ce moyen diagnostique [3]. Le contenu luminal est liquide, jaune paille, ou crémeux épais, plus blanc. La kératine est présente en quantités variables. En cas d'inflammation, des cristaux de cholestérol et des corps hyalins sont présents. Quand il y a inflammation de la paroi kystique, l'épithélium se transforme progressivement de manière significative en un épithélium non kératinisé assez similaire à celui des kystes odontogènes inflammatoires banals. Le diagnostic différentiel doit être posé avec les autres kystes odontogènes, avec les kystes fissuraires, avec l'améloblastome, voire avec le fibrome ou le myxome odontogéniques. Le traitement est chirurgical. L'exérèse complète peut être difficile parce que la paroi du kyste est mince et fragile, parce que le kyste est multiloculaire, parce qu'il a des cavités satellites ou parce que sa localisation est profonde. La rupture des corticales osseuses et l'infec- 
tion rendent aussi l'exérèse complète difficile. Une fixation chimique après l'énucléation par une solution de Carnoy permettait de diminuer le taux de récidives; son usage n'est plus autorisé en Europe. Le kératokyste a un taux de récidive élevé, qui varie entre 3 et $60 \%$ selon les séries [1]. Il est suggéré que la technique opératoire d'exérèse joue un rôle important dans le degré de récidive. Les contrôles radiographiques réguliers doivent s'étaler sur au moins 5 ans. Des cas de dégénérescence maligne ont aussi été rapportés [4].

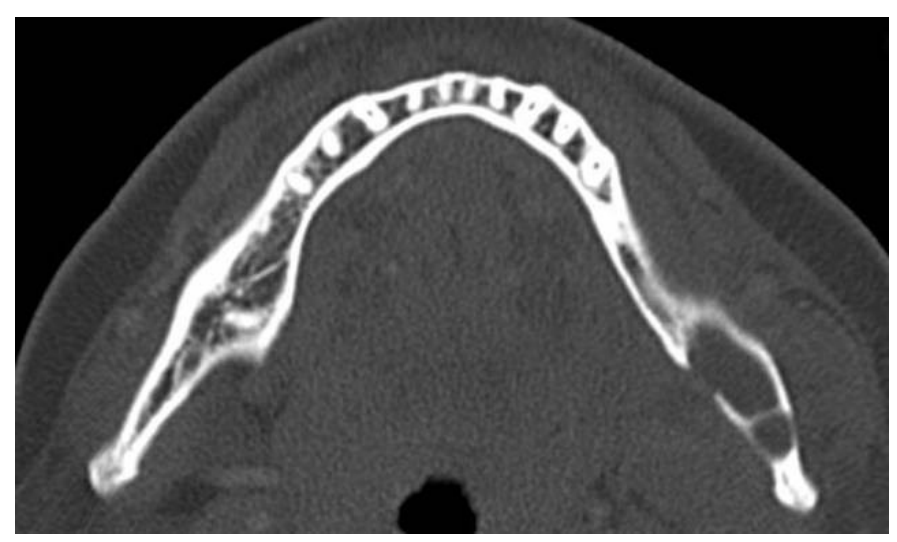

Fig. 4. CT scan axial mandibulaire: kératokyste multiloculaire angulaire droit.

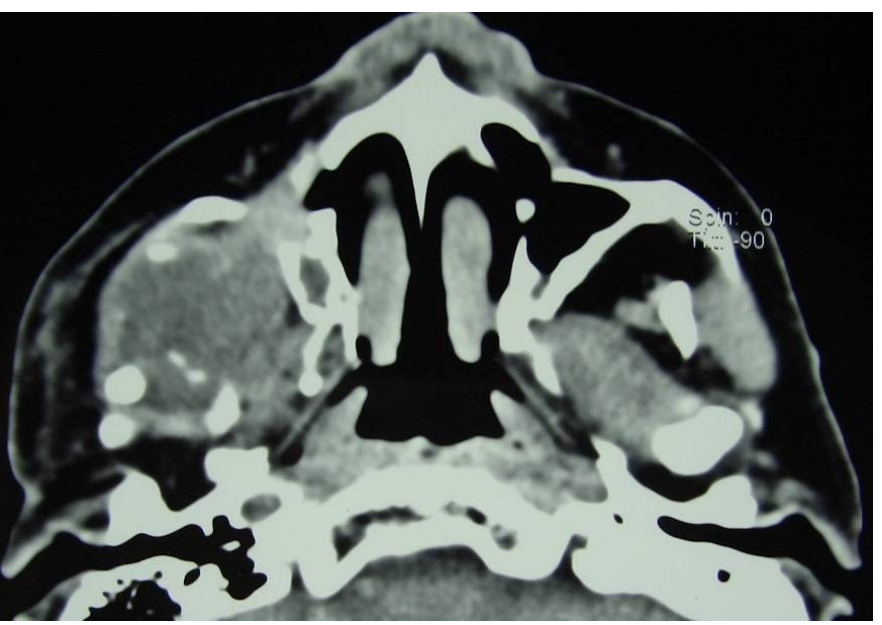

Fig. 5. Kératokyste de la partie postérieure du maxillaire supérieur droit chez un patient atteint du syndrome de Gorlin-Goltz. 


\section{Améloblastome}

C'est une tumeur de l'organe de l'émail dont la différenciation ne va pas jusqu'à la formation d'émail. Le terme "améloblastome" est donc mal choisi dans la mesure où la tumeur ne dérive pas des améloblastes. L'améloblastome peut prendre naissance à partir de restes cellulaires de l'organe de l'émail, de cellules de l'organe de l'émail en développement, de cellules épithéliales des kystes odontogènes (en particulier les kystes folliculaires), de cellules basales de l'épithélium de surface des mâchoires ou d'épithélium hétérotopique en d'autres endroits de l'organisme (hypophyse, os longs).

L'améloblastome central (intra-osseux) est fréquemment associé avec une dent incluse et/ou un kyste folliculaire [5]. L'améloblastome périphérique (extra-osseux) est originaire soit de l'épithélium de surface soit de restes de la lame dentaire; il se développe dans les tissus mous qui recouvrent les procès alvéolaires [6]. Cliniquement, l'améloblastome central intra-osseux survient à tout âge, l'âge moyen de découverte étant de 33 ans (50\% des cas surviennent entre 20 et 40 ans). La distribution entre les sexes est la même, mais on note une prédominance dans la race noire. La localisation est le plus souvent mandibulaire (environ $80 \%$ des cas), généralement au niveau de la branche horizontale, en région molaire, et au niveau de l'angle (environ $75 \%$ des cas) (Figure 6). La croissance est généralement lente, avec une soufflure osseuse progressive de l'os et un amincissement des corticales. Les douleurs ne surviennent souvent qu'en cas d'infection. Les dents sont très fréquemment mobilisées en raison de leur résorption radiculaire. Radiologiquement, l'améloblastome central apparaît initialement comme une lacune uniloculaire bien délimitée. Rapidement, ce processus ostéolytique central (charnu au CT scan) devient multiloculaire et souffle l'os, amincissant les corticales (Figures 8-9). Les cloisons de refend sont alors habituelles; c'est l'aspect typique en «bulles de savon ». Il n'est pas rare de trouver une dent incluse. Les résorptions radiculaires sont habituelles. La IRM se révèle très utile dans l'évaluation des récidives, grâce à ses possibilités de différenciation tissulaire sur base des compositions protoniques. Le diagnostic différentiel se pose le plus souvent avec le kyste folliculaire, le kératokyste, le fibrome améloblastique, le fibrome odontogénique, le myxome odontogénique ou avec le granulome central de réparation à cellules géantes. Le traitement est chirurgical : c'est l'exérèse large et complète [7]. La tumeur étant localement agressive et infiltrante dans l'os spongieux, elle présente une tendance marquée à la récidive. C'est pourquoi le curetage simple ne peut pas être recommandé comme traitement. Une marge de résection de sécurité est donc nécessaire et une surveillance à long terme est indispensable. Le taux de récidive avoisine $75 \%$ (Figure 7) avec les traitements chirurgicaux conservateurs et seulement $15 \%$ avec les traitements plus radicaux. 


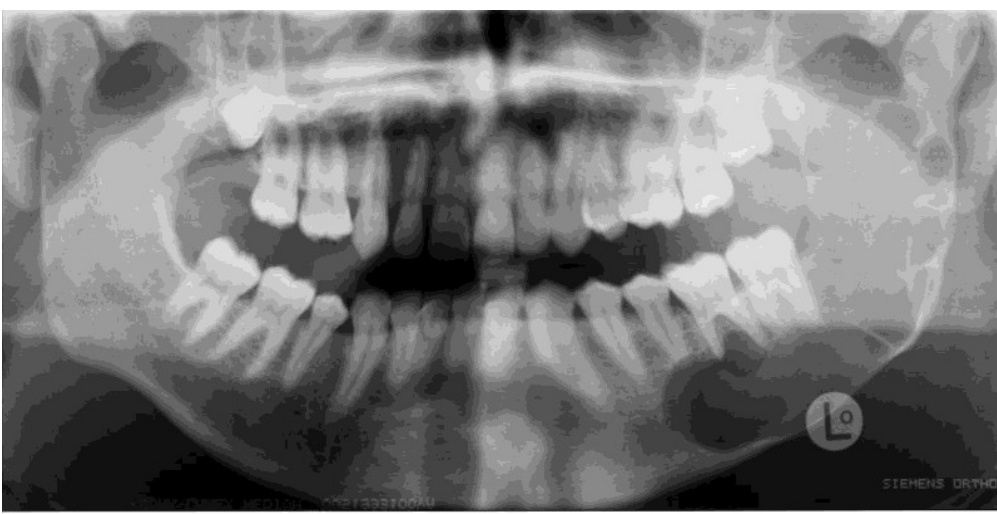

Fig. 6. Améloblastome branche montante et angle mandibulaire gauche.

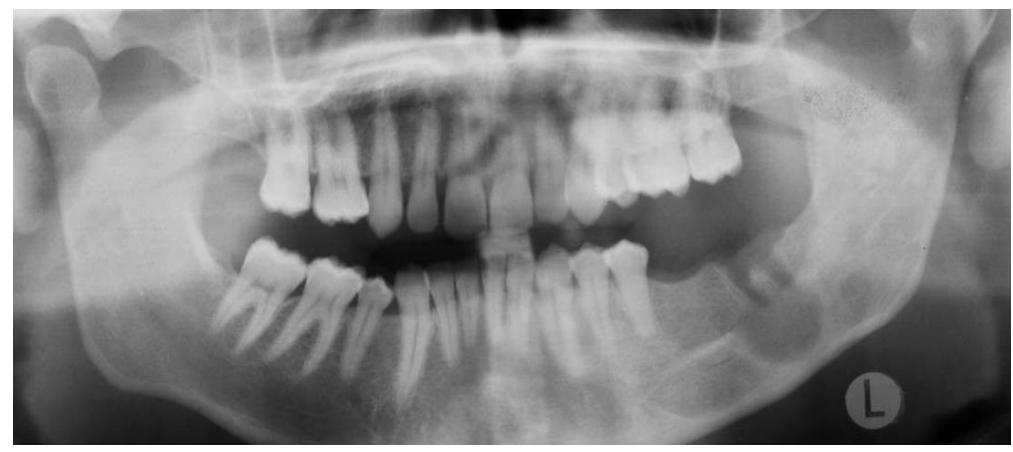

Fig. 7. Récidive 18 mois après exérèse macroscopiquement complète. 


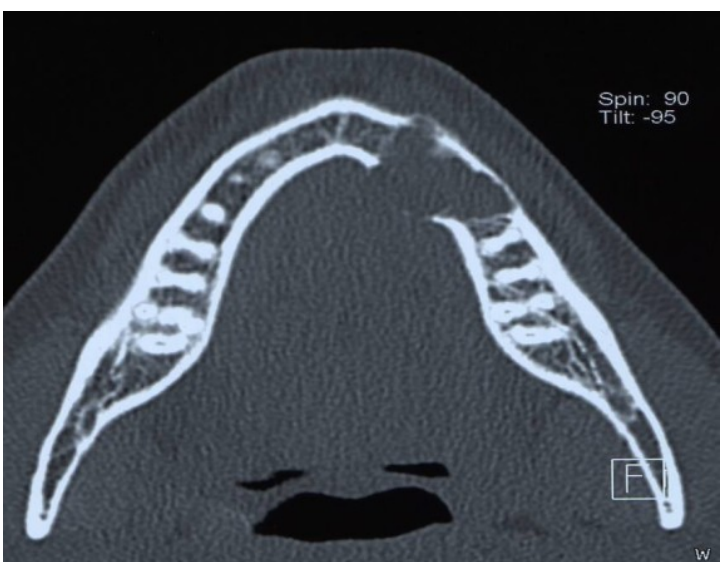

Fig. 8. CT axial mandibulaire: améloblastome de la partie antérieure de la branche horizontale, avec rupture de la corticale interne.

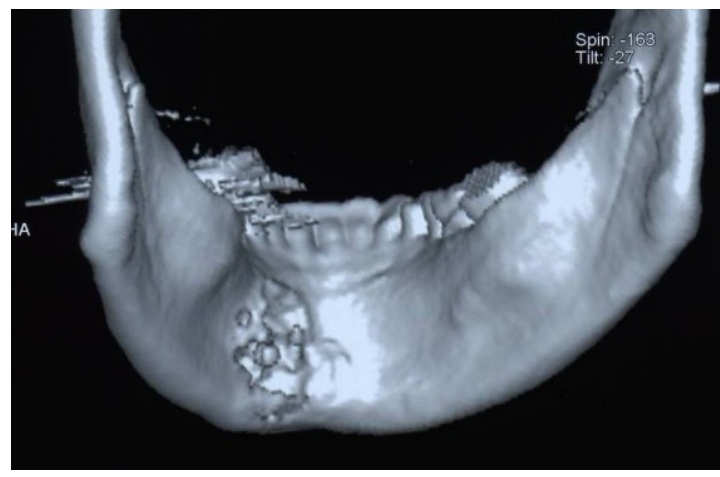

Fig. 9. Rupture de la corticale linguale bien visualisée sur cette reconstruction $3 \mathrm{D}$.

216 

de Pindborg)

Tumeur rare, elle affecte surtout l'individu adulte d'âge moyen. Les deux sexes sont affectés de la même manière. La tumeur a une prédilection pour la mandibule (Figure 10), particulièrement en région prémolaire et molaire et provient de restes de sac folliculaire ou de restes épithéliaux parodontaux [8]. Cliniquement, la tumeur de Pindborg est le plus souvent asymptomatique. Dans la moitié des cas, une association avec une dent incluse est retrouvée. Radiologiquement, la tumeur apparaît souvent comme une ostéolyse bien limitée (Figure 11); néanmoins, l'aspect peut être très variable : soit aspect uniloculaire radio-clair bien limité, soit aspect multiloculaire, soit aspect diffus avec alternance centrale de zones radioclaires et de zones opaques calcifiées. Les masses radio-opaques sont souvent en relation avec la couronne d'une dent incluse [9]. Le diagnostic différentiel se pose avec le kyste folliculaire, avec le kératokyste, avec le kyste odontogène calcifiant, avec le myxome odontogène, le granulome à cellules géantes et l'améloblastome. Le traitement consiste en l'exérèse complète avec si nécessaire curetage osseux appuyé. Le taux de récidive est bas.

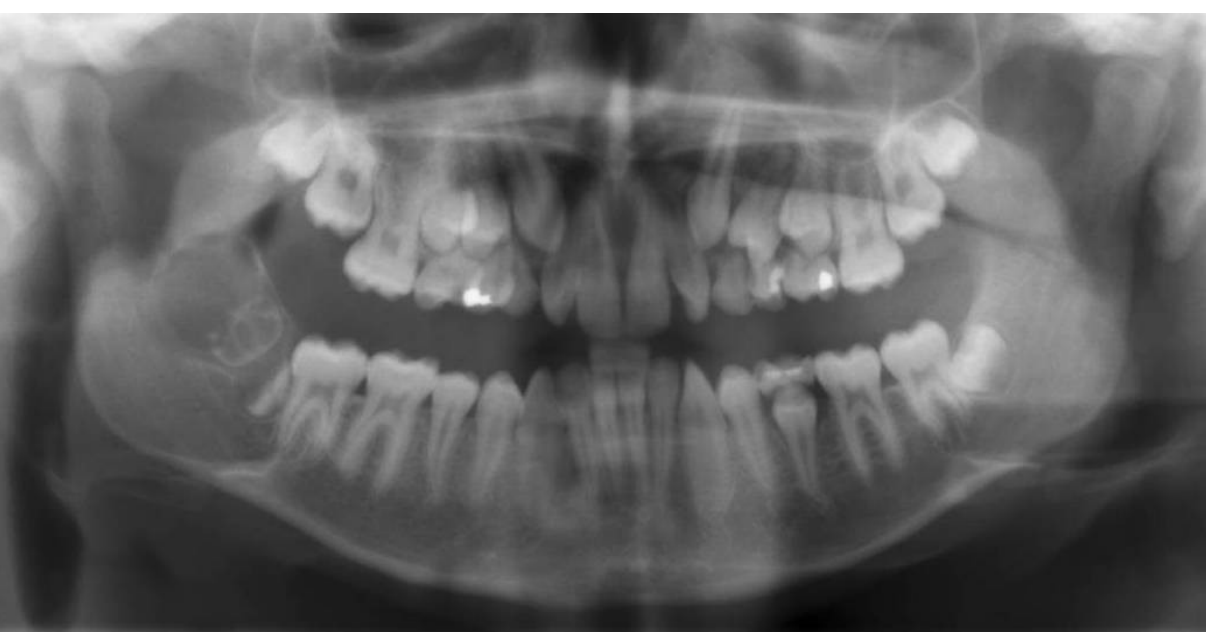

Fig. 10. Tumeur de Pindborg de la branche montante droite. 


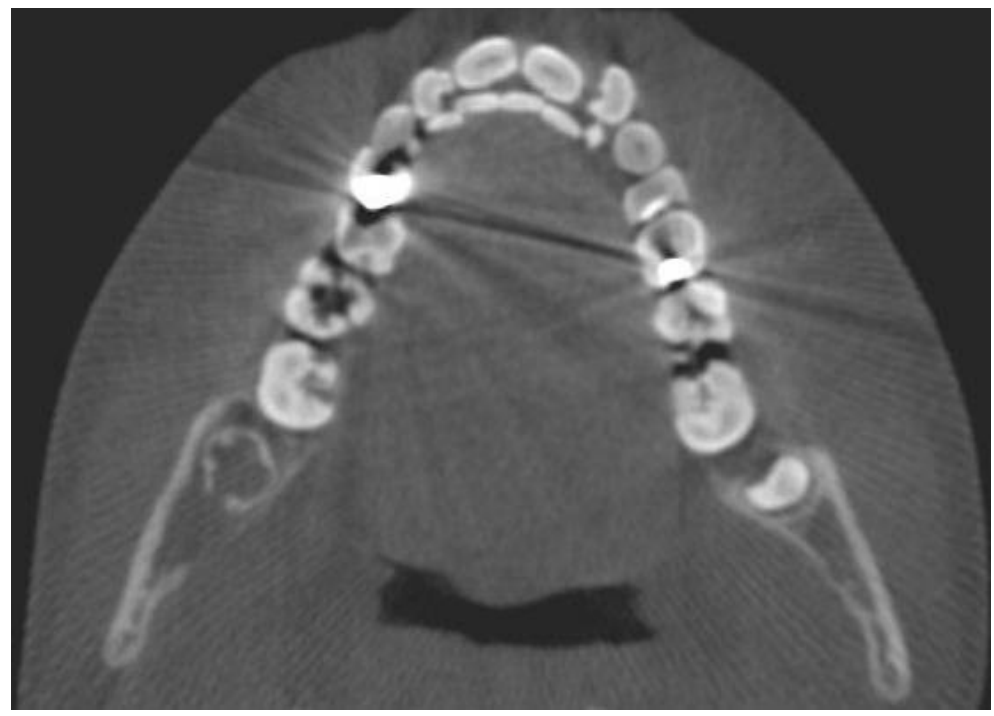

Fig. 11. CT scan axial mandibulaire de la même tumeur de Pindborg.

\section{Fibrome améloblastique}

Cette tumeur est caractérisée par la prolifération simultanée d'un tissu épithélial odontogène et d'un tissu mésenchymateux semblable à celui de la papille dentaire mais sans la couche des odontoblastes. Elle se formerait aux dépens des cellules épithéliales de la gaine de Hertwig, qui exercent des effets inducteurs sur le mésenchyme avoisinant. Le fibrome améloblastique représente environ $2 \%$ des tumeurs odontogéniques. Il survient chez le sujet jeune, près de 20 ans avant l'âge de survenue de l'améloblastome. La distribution est presque égale chez l'homme et chez la femme et il n'y a pas de prépondérance raciale. La localisation est essentiellement mandibulaire, en région prémolaire-molaire [10]. Cliniquement, la croissance est généralement lente et indolore. Les tumeurs de grande taille déplacent et mobilisent les dents et engendrent des ulcérations muqueuses douloureuses et hémorragiques. Radiologiquement, la tumeur apparaît comme une géode souvent uniloculaire, à bords nets (Figure 12). Plus grosse, elle devient multiloculaire, soufflant les corticales sans cependant les envahir; les cloisons de refend sont habituelles. Les racines dentaires sont déplacées et éventuellement résorbées. Des dents incluses peuvent être associées. Le diagnostic différentiel doit être fait avec les autres kystes et tumeurs odontogènes, avec le kyste osseux anévrismal, avec le granulome central de réparation à cellules géantes, ou avec l'ostéose fibrokystique de Von Recklinghau- 
sen. Le traitement consiste en l'exérèse chirurgicale; des récidives peuvent survenir. Une surveillance radiologique à long terme est recommandée, des cas de transformation en fibrosarcome améloblastique ayant été décrits [11].

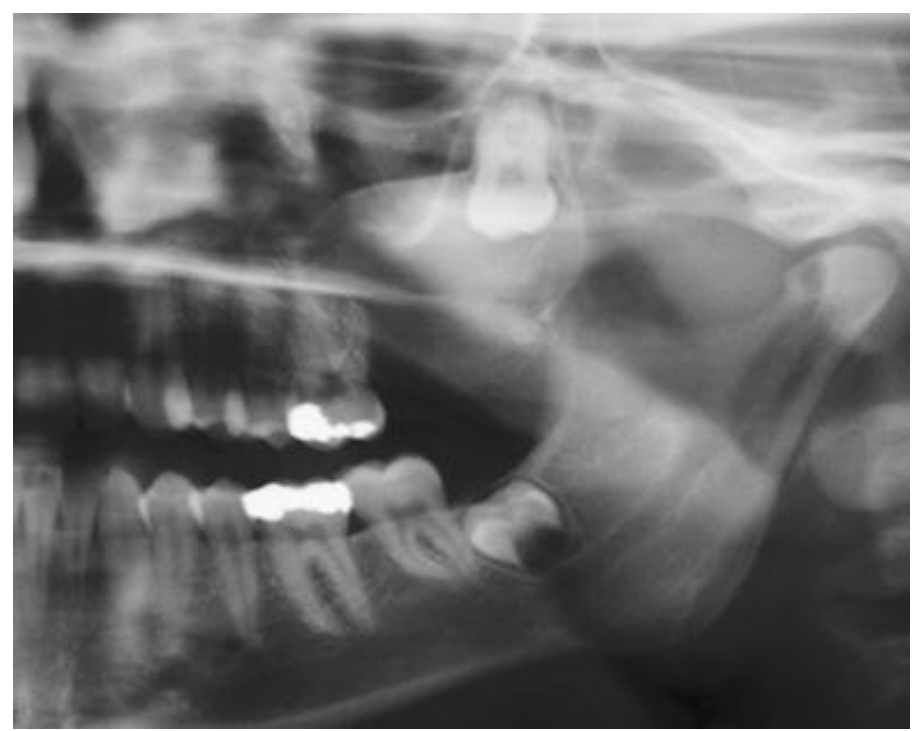

Fig. 12. Fibrome améloblastique autour de la dent $n^{\circ} 28$ incluse.

\section{Fibrosarcome améloblastique}

C'est la variété maligne du fibrome améloblastique [12], dans laquelle la composante mésenchymateuse a dégénéré et l'épithélium odontogène est resté bénin. Cette tumeur est extrêmement rare et affecte les adultes jeunes; la localisation est plus souvent mandibulaire [11]. Cliniquement, la croissance est rapide et douloureuse, avec destruction osseuse, ulcérations de surface et mobilisation des dents; le signe de Vincent d'Alger est habituel. Radiologiquement, l'image d'un processus expansif malin est présente : ostéolyse irrégulière mal délimitée avec soufflure du maxillaire et érosion corticale. Le diagnostic différentiel se pose avec le fibrome améloblastique, avec l'améloblastome, avec les autres tumeurs malignes odontogènes ou non. Le traitement est chirurgical de type oncologique. Le taux de récidive est élevé et le pronostic est pauvre. 


\section{Myxome odontogénique}

C'est une tumeur très rare qui se développe à partir de la composante mésenchymateuse du germe dentaire, soit au niveau de la papille dentaire, soit au niveau du follicule, soit encore au niveau du ligament desmodontal. La tumeur survient chez l'adulte jeune et affecte également les deux sexes [13]. Cliniquement, la tumeur est centrale et a une croissance lente, le plus souvent asymptomatique. Elle se manifeste par une voussure osseuse; les dents peuvent être déplacées et mobilisées. Radiologiquement (Figure 13), la lacune expansive est destructive et a un aspect moucheté ou "en rayons de miel"; elle peut aussi être uni- (33\% des cas) [14] ou multiloculaire (55\% des cas) [14]. Les résorptions radiculaires ne sont pas fréquentes. Le diagnostic différentiel doit être fait avec l'améloblastome, avec les dysplasies fibreuses, avec le fibrome odontogénique, avec le myxofibrome odontogénique, avec le neurofibrome myxoїde, avec le fibrome chondromyxoïde, avec le granulome central de réparation à cellules géantes ou avec le kyste osseux anévrysmal. Le traitement consiste en l'exérèse chirurgicale. Les récidives sont fréquentes, ce qui impose une surveillance radiologique.

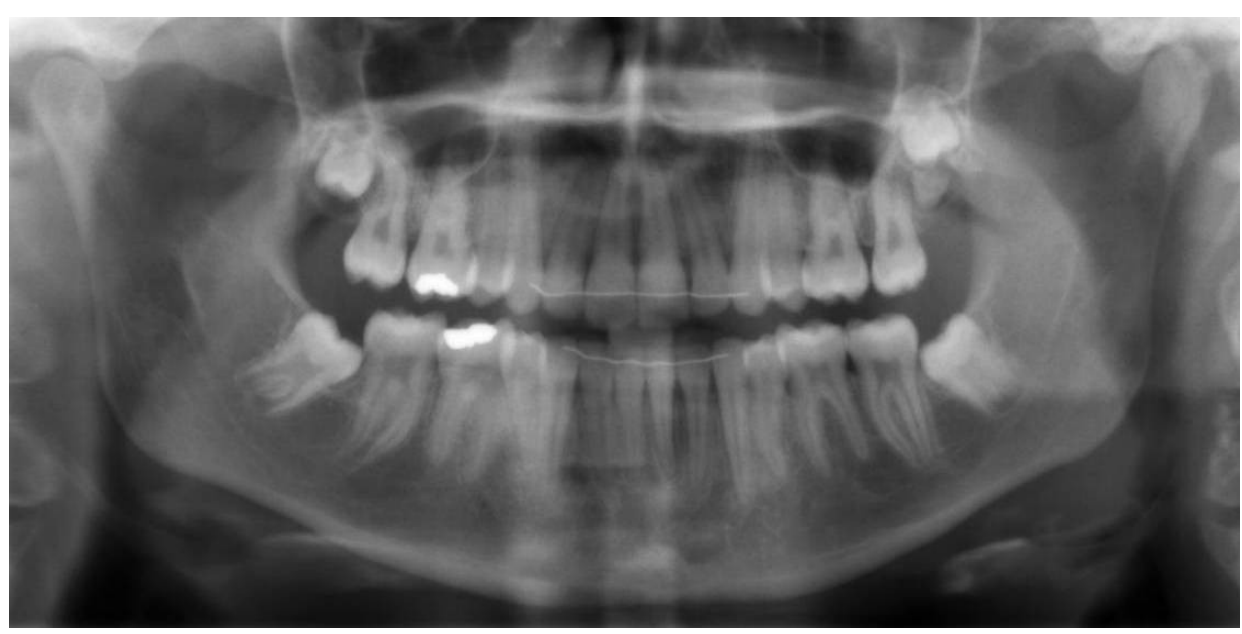

Fig. 13. Myxome au niveau de la dent $n^{\circ} 28$. 


\section{Carcinome primitif intra-osseux}

Appelée aussi "carcinome épidermoïde primitivement intra-alvéolaire", cette tumeur maligne peut se développer à partir de restes épithéliaux odontogènes intraosseux, à partir de restes de Malassez ou encore à partir de cellules épithéliales enclavées lors de la fusion des bourgeons embryonnaires. Cette tumeur doit donc être distinguée des carcinomes épidermoïdes muqueux et des carcinomes métastatiques. Cette tumeur est diagnostiquée plutôt chez l'homme que chez la femme, entre les sixième et septième décennies, et se localise préférentiellement à la mandibule [15]. Cliniquement, les symptômes sont ceux d'un processus malin rapidement expansif, avec tuméfaction inflammatoire, algies, mobilités dentaires et ulcération en surface. L'aspect radiologique n'est pas caractéristique (Figures 14-16); c'est celui d'une tumeur maligne osseuse, avec radioclarté diffuse [16]. Le diagnostic différentiel est celui des tumeurs malignes à développement osseux. Le traitement consiste en l'exérèse large, avec évidement ganglionnaire. Le pronostic à 5 ans oscille entre 30 et 40 $\%$.

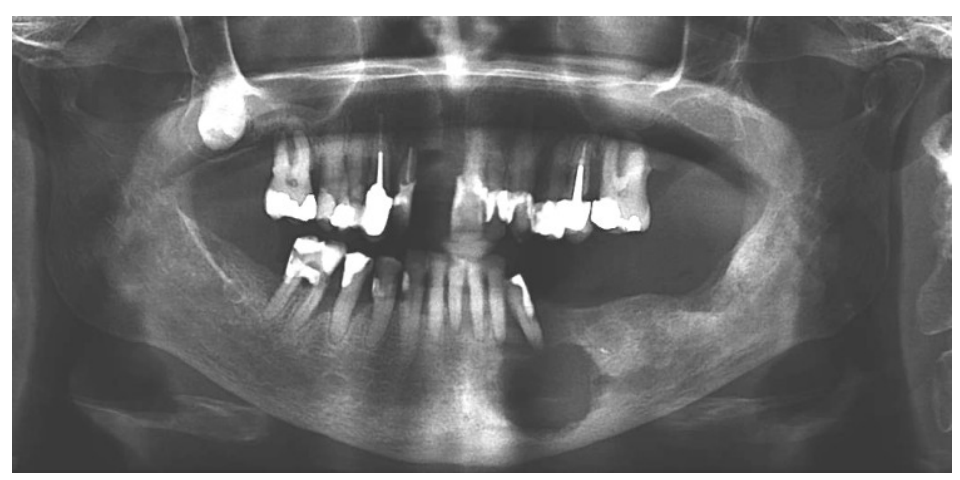

Fig. 14. Carcinome odontogène intra-osseux de la branche mandibulaire horizontale gauche, se présentant comme une image « kystique » mais avec troubles de la sensibilité dans le territoire du nerf alvéolaire inférieur gauche. 


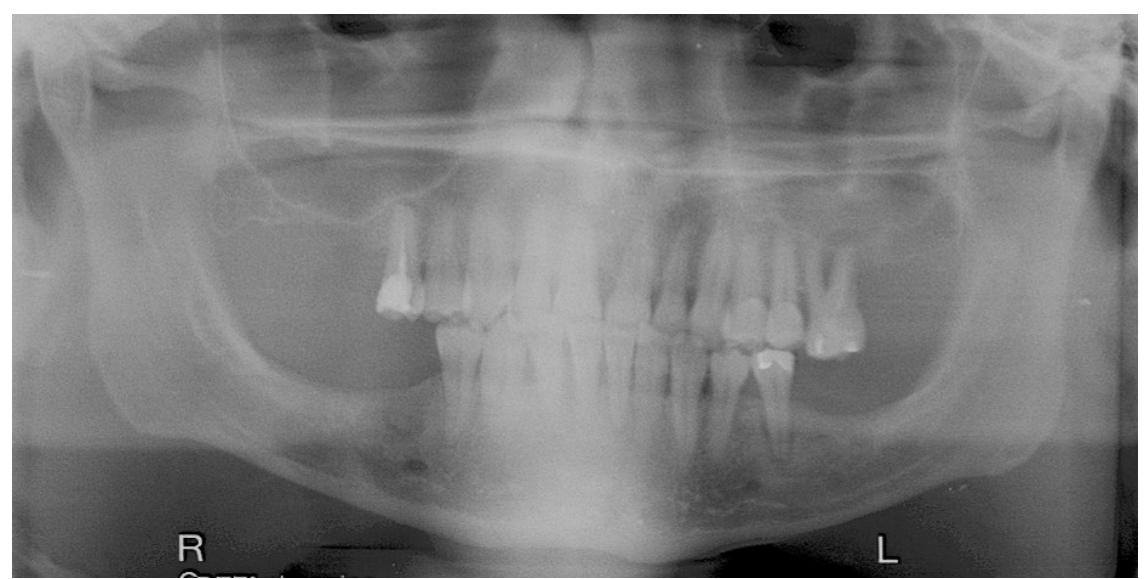

Fig. 15. Carcinome primitif intra-osseux apparu cliniquement comme une parodontite sur la 35 , mais avec troubles de la sensibilité dans le territoire du du nerf alvéolaire inférieur gauche.

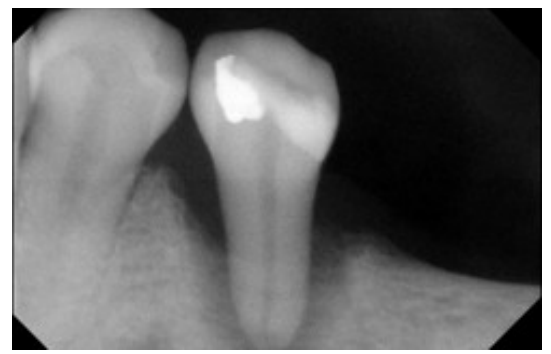

Fig. 16. Radiographie apicale, dent $n^{\circ} 35$.

\section{Fibrome cémento-ossifiant}

Le fibrome cémentifiant partage beaucoup de caractéristiques avec le fibrome ossifiant; des formes hybrides ou "fibromes cémento-ossifiants" existent, qui pourraient n'être d'ailleurs que des variétés potentiellement agressives des fibromes ossifiants, lésions distinctes des dysplasies fibreuses. La tumeur peut survenir à tout âge, affecte plus fréquemment la femme que l'homme. La mandibule est trois fois plus atteinte que les maxillaires supérieurs [17]. Cliniquement, la croissance est centrifuge, lente et asymptomatique; la tumeur est découverte quand le galbe osseux est défor- 
mé. Les dents peuvent être déplacées précocement; elles restent vitales. Radiologiquement (Figures 17-19), l'aspect est variable en fonction du stade de développement [18]. Au début, la tumeur apparaît comme une lacune arrondie éventuellement polylobée, qui se délimite nettement par rapport à l'os environnant. Plus tard, des opacités apparaissent dans la radioclarté; ces opacités confluent progressivement et peuvent finalement former une masse importante très dense. Les corticales osseuses sont soufflées et amincies et la tumeur garde un aspect arrondi. Les résorptions radiculaires sont inhabituelles. Le diagnostic différentiel doit être posé avec la dysplasie périapicale du cément, avec le cémentoblastome bénin, avec le fibrome ossifiant, avec les dysplasies fibreuses de l'os, avec l'ostéite condensante ou avec l'ostéome ostéoïde. Le traitement consiste en l'énucléation chirurgicale de la tumeur. Les récidives $(12 \%)$ surviennent en raison de la difficulté de l'exérèse complète, surtout dans les grandes lésions [19].

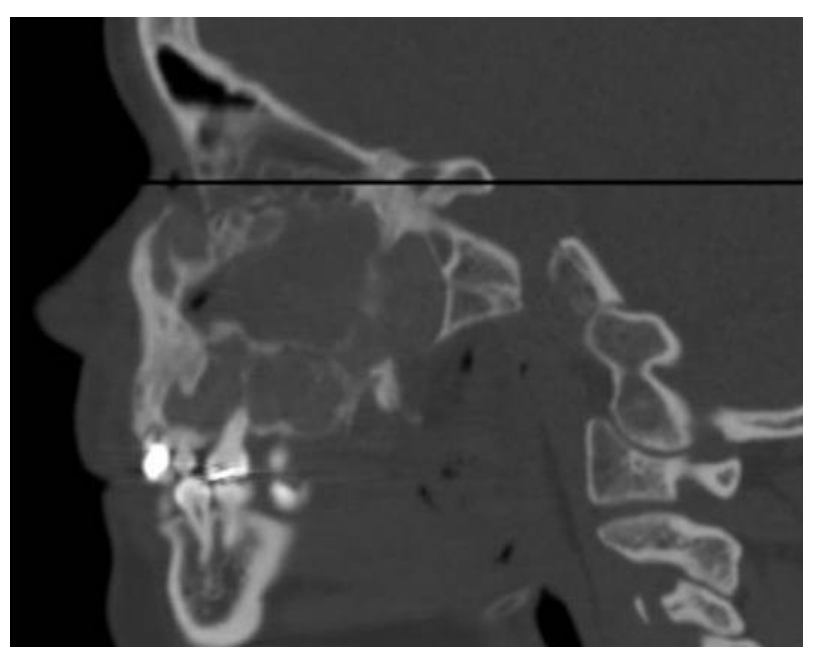

Fig. 17. Fibrome cémentifiant du maxillaire supérieur, CT scan sagittal paramédian. 


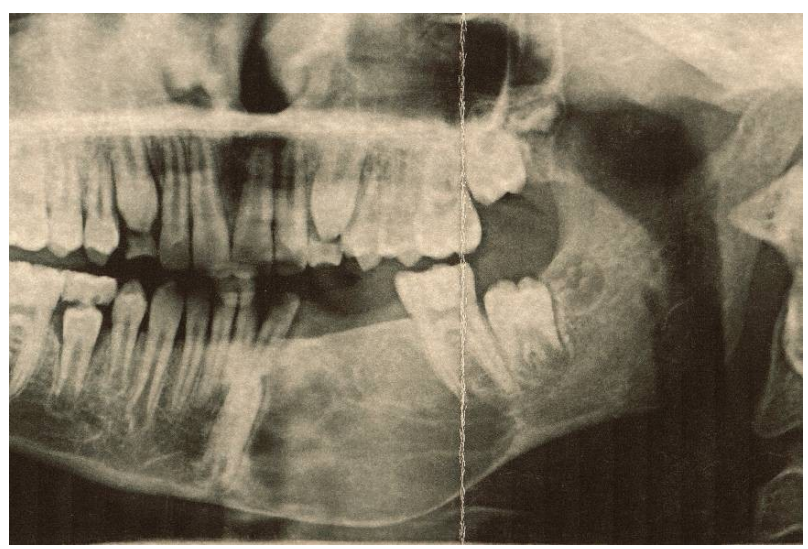

355

356

357
Fig. 18. Fibrome cémentifiant de la branche horizontale gauche de la mandibule.

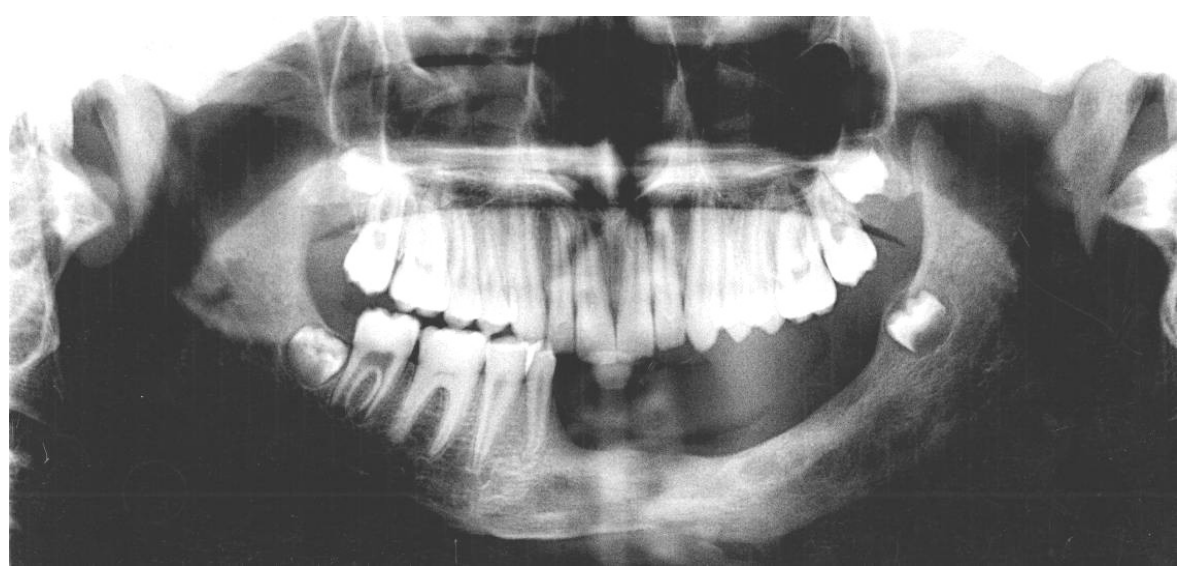

Fig. 19. Résultat à un an après résection et greffe osseuse iliaque libre (voir Figure 18). 


\section{Dysplasie périapicale du cément}

Cette lésion est considérée comme un processus réactionnel périapical (à des microtraumatismes chroniques?). La lésion affecte plus souvent la femme que l'homme, presque toujours après l'âge de 20 ans; la lésion touche plus fréquemment la race noire. La localisation est dans deux tiers des cas mandibulaire, généralement en région incisive, parfois en région prémolaire ou molaire. Les lésions, habituellement multiples et cliniquement asymptomatiques, se développent dans ou au voisinage du ligament desmodontal, en région apicale. La vitalité des dents affectées est conservée. La découverte est généralement radiologique, accidentelle (Figures 20, 21). Au début, l'aspect est ostéolytique : il existe une lacune périapicale due à la fibrose avec ostéolyse locale. Ensuite, avec le dépôt de cément, la radioclarté devient progressivement radio-opaque en son centre. Les opacités confluent pour former une masse unique qui reste cependant entourée par une image de bordure radio-claire [20]. Le diagnostic différentiel est fait au début avec le granulome apical. Plus tard, à la phase de maturité, le diagnostic se pose avec le cémentoblastome bénin, avec l'ostéite condensante, avec l'ostéomyélite chronique focale sclérosante, avec l'ostéome ostéoïde ou avec l'ostéoblastome. Le traitement n'est généralement pas nécessaire, on peut se contenter de surveiller l'évolution de la lésion par des contrôles radiologiques.

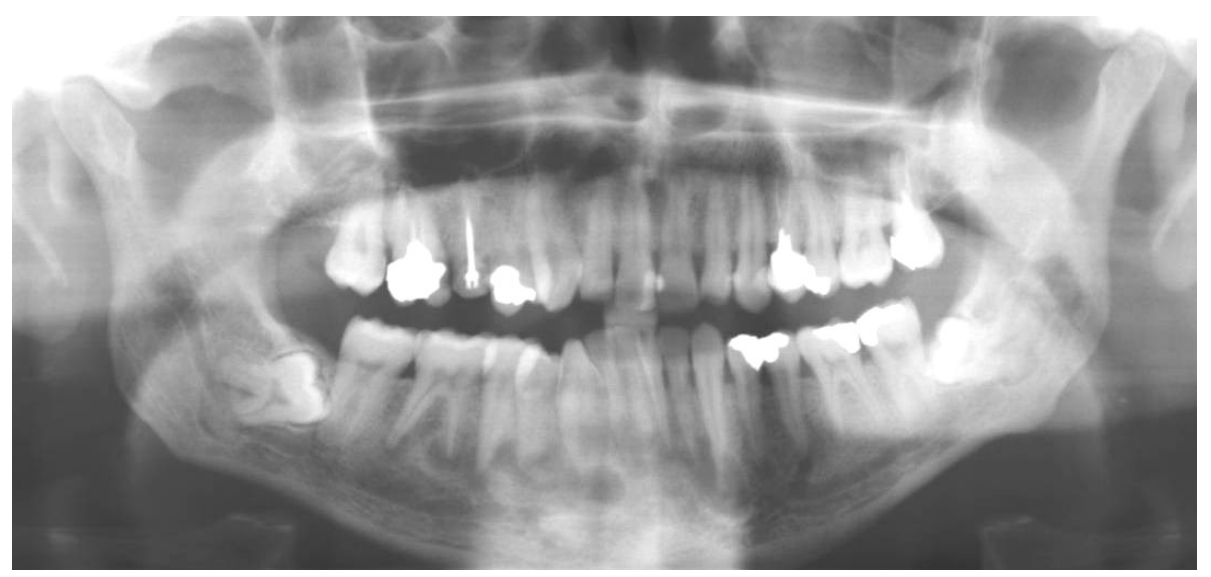

Fig. 20. Orthopantomogramme montrant des radioclartés apicales au niveau des incisives et des molaires inférieures, chez un sujet de race noire : dysplasie périapicale du cément. 


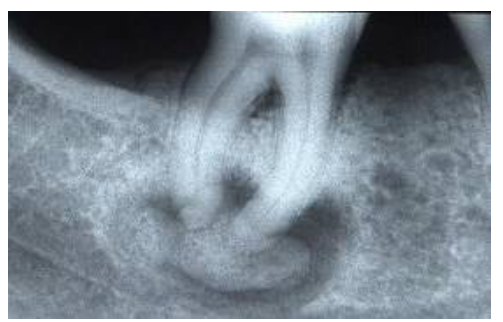

Fig. 21. Radioclarté périapicale sur une dent vitale, avec une radio-opacité centrale, ayant la densité radiologique du cément.

\section{Kyste osseux anévrismal}

C'est une dystrophie osseuse pseudo-tumorale, bénigne et habituellement solitaire, qui réalise une cavitation intra-osseuse uni- ou pluriloculaire à contenu hématique. L'incidence est très faible: 1 à $4 \%$ des tumeurs et dystrophies bénignes osseuses. Plus de $70 \%$ des patients sont âgés de moins de 20 ans; les femmes sont un peu plus souvent affectées que les hommes. La localisation mandibulaire est la plus fréquente, surtout au niveau de l'angle et de la branche horizontale [21]. L'étiopathogénie est le plus souvent considérée comme de nature vasculaire : un «shunt» artérioveineux serait développé à partir d'une lésion osseuse primitive et entraînerait une lésion osseuse secondaire, de nature réactionnelle. Ceci explique l'association fréquente avec d'autres tumeurs osseuses bénignes comme le granulome gigantocellulaire et la dysplasie fibreuse. Certains auteurs ont invoqué une étiologie traumatique ou tumorale, d'autres un trouble du développement osseux. Cliniquement, une tuméfaction très souvent indolore, d'évolution relativement lente, est responsable d'asymétrie faciale; il n'y a pas d'altération neurologique périphérique et la vitalité des dents, refoulées par le processus à croissance lente, est préservée. La corticale osseuse est rarement rompue. Radiologiquement (Figure 22), l'ostéolyse circonscrite a un aspect réticulé, avec des cloisons osseuses en «nid d'abeilles» ou «bulles de savon», et est bordée par un fin liseré d'ostéocondensation. La corticale osseuse peut être soufflée; la résorption radiculaire dentaire est occasionnelle. Le diagnostic différentiel radiologique doit être fait avec l'hémangiome, le granulome éosinophile, le myxome, la tumeur à cellules géantes et certaines tumeurs lytiques odontogéniques. Le diagnostic est anatomo-pathologique. Macroscopiquement, la lésion osseuse est de coloration bleutée; l'os est remplacé par du tissu fibreux et spongieux. Les cavités sont remplies de sang. L'évolution est bénigne. Le traitement de choix est le curetage qui est parfois très hémorragique. Comme plus de la moitié des cas récidivent, une surveillance radiologique s'impose [22]. 


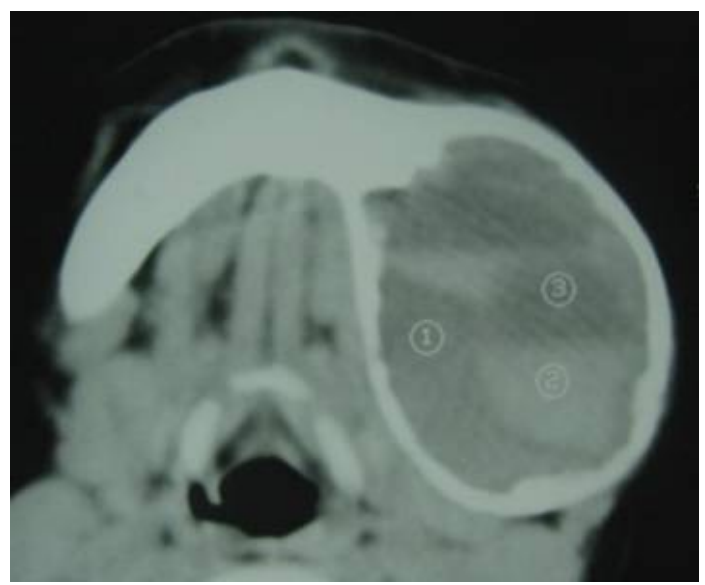

Fig. 22. CT scan axial mandibulaire illustrant une kyste anévrysmal mandibulaire.

\section{Ostéoradionécrose}

Il s'agit d'une nécrose osseuse aseptique post-radiothérapique due à l'impossibilité pour les ostéoblastes à remplir leur fonction ostéogénique, suite principalement à un trouble vasculaire. L'os irradié subit principalement une ischémie, une dévascularisation responsable d'une hypoxie cellulaire [23]. Sur ce terrain dévascularisé survient alors soit spontanément soit secondairement à un (micro)traumatisme, une perte de substance osseuse (et muqueuse) et une surinfection. L'intensité des agents ionisants (dose et durée) est prépondérante mais une dose tumoricide est malheureusement toujours à haut risque d'ostéoradionécrose. Les traumatismes déclenchant cette complication sont le plus souvent dentaires : extraction, soin parodontal, soin endodontique, trauma prothétique. La mise en place d'implants est également un facteur de risque non négligeable. Cette ostéoradionécrose ne survient qu'à la mandibule, en raison de la vascularisation toute différente au maxillaire supérieur et en raison de la structure plus spongieuse que corticale à ce niveau (Figure 23). Les symptômes ne sont pas spécifiques, ils sont semblables à toute ostéomyélite : douleur importante, trismus, fracture spontanée, cellulite, retard de cicatrisation, formation de séquestres osseux avec découverte de l'os et perte de substance importante (orostome). Le traitement est lourd et difficile : il fait appel à des mesures médicales (antibiothérapie massive dirigée et de longue durée) et chirurgicales (résection/greffe ou curetage localisé). Souvent, cela mène à des pertes de substance bien 
plus difficiles à traiter que celles résultant de la résection tumorale. La prophylaxie est dès lors capitale [24].

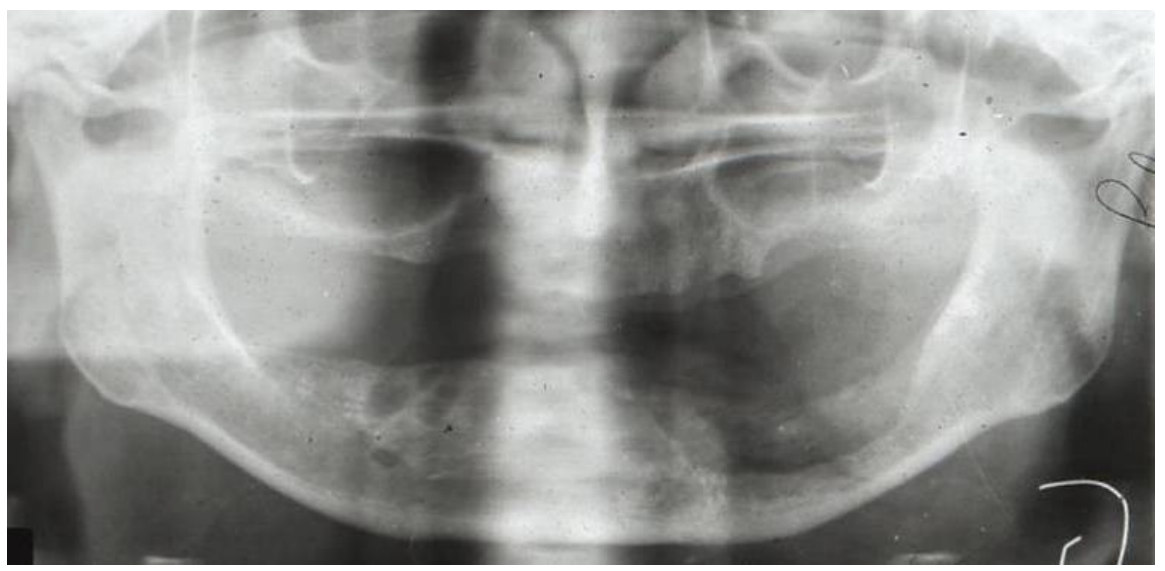

Fig. 23. Orthopantomogramme montrant une ostéolyse térébrante de la branche horizontale gauche de la mandibule, après radiothérapie, évoquant une ostéoradionécrose.

\section{Ostéonécrose des maxillaires chez patients traités par des biphosphonates}

Ces dernières années est apparu un tableau clinique ressemblant très fort à celui de l'ostéoradionécrose, chez des patients traités aux biphosphonates (BPO) prescrits pour prévenir ou traiter des pathologies osseuses ostéolytiques [25]. On doit distinguer les BPO administrés par voie orale, pour traiter principalement l'ostéoporose et d'autres affections osseuses ostéolytiques, et les BPO administrés par voie iv pour traiter des métastases osseuses, le myélome multiple et d'autres maladies ostéolytiques invalidantes (fractures, tassement vertébral...). Par définition, il s'agit d'une ostéonécrose des maxillaires, survenant spontanément ou le plus souvent après un acte chirurgical au niveau des maxillaires, sans autre cause évidente que l'administration de BPO. Cette ostéonécrose se présente essentiellement mais pas uniquement à la mandibule, et touche plus particulièrement les hommes atteints de myélome multiple, mais aussi les femmes traitées par BPO intraveineux pour métastases osseuses. Les patient(e)s rhumatologiques traité(e)s aux BPO oraux ne sont quasi jamais atteints mais le risque n'est cependant pas nul. L'ostéonécrose résulte d'une toxicité pour les cellules épithéliales, d'une inhibition de l'ostéoclasie et d'une 
diminution de l'angiogenèse. Il ya toujours une infection osseuse, qu'elle soit surajoutée ou causale reste à démontrer. Des actinomycètes sont toujours présents dans le tissu osseux nécrotique, mais cela ne préjuge en rien de son éventuel rôle inducteur de l'ostéonécrose. Les signes cliniques sont des douleurs, des tuméfactions inflammatoires ou infectieuses, des déhiscences muqueuses (parfois impressionnantes) laissant apparaître des surfaces osseuses dénudées, des fistules, des fractures spontanées. Le dosage du CTX (C-télopeptide) comme témoin du métabolisme osseux est inutile et non validé, car tout patient traité par BPO l'est pour une pathologie ostéolytique responsable d'une diminution du CTX [26]. Les signes radiologiques ne sont pas typiques (Figure 24): une ostéolyse mal circonscrite, des fractures spontanées ou encore des signes d'apposition osseuse (réactionnelle). Le diagnostic est simple si on fait le lien avec la prise de BPO, après exclusion des autres causes infectieuses et/ou tumorales. Le pronostic semble sombre quoique le recul manque pour l'établir. L'évolution paraît souvent inexorable, menant à des surinfections, des complications fonctionnelles, des séquestres et des destructions osseuses qui se poursuivent souvent d'autant plus vite qu'on intervient dans la zone nécrotique. La guérison survient rarement. Le traitement est méconnu et décevant [27]. Les antibiotiques sont réservés aux surinfections, les anti-inflammatoires non-stéroïdiens (AINS) et l'oxygénothérapie hyperbare sont inefficaces. Les antibiotiques à prescrire sont des dérivés de la pénicilline et chez les patients allergiques, du métronidazole associé à des quinolones ou des macrolides. La clindamycine n'est pas recommandée, en raison de sa plus faible efficacité sur les Actinomycètes régulièrement retrouvés dans les zones d'ostéonécrose. Les bains de bouche antiseptiques sont utiles au maintien d'un état de propreté au sein de la cavité orale. Il semble que tout geste chirurgical aussi mineur qu'il soit (curetage par exemple) induise une extension de la destruction osseuse. Aussi, tout acte «chirurgical» oral sera réalisé chez les patients traités par BPO iv sous couverture antibiotique; les BPO oraux seront arrêtés avant tout acte chirurgical, si possible trois mois. Il n'est pas prouvé que l'arrêt des BPO permette la guérison des lésions osseuses des maxillaires. Il est utile de référer les patients au spécialiste, faute de recommandations bien précises actuellement. 


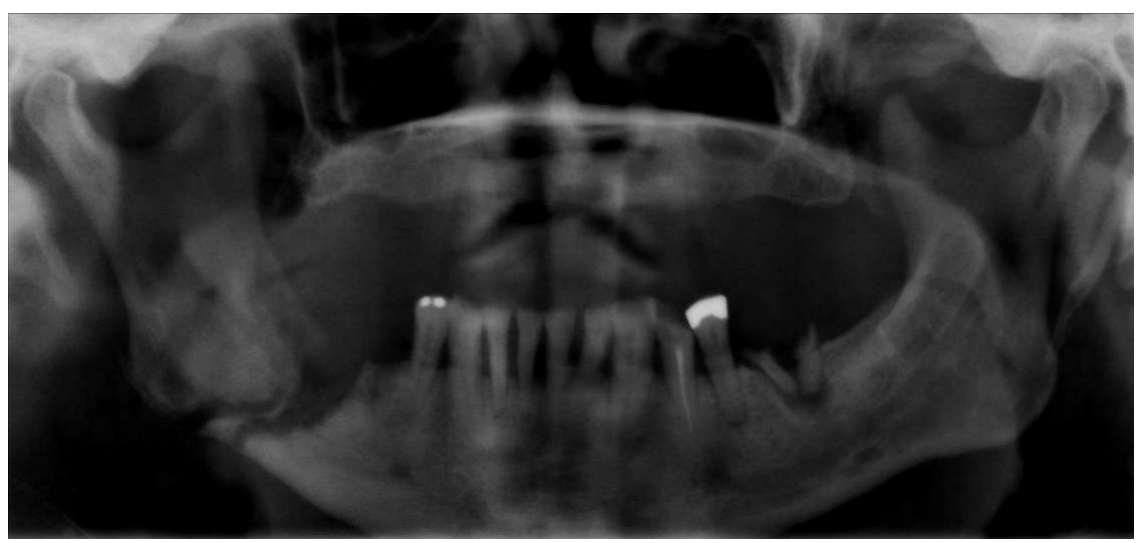

Fig. 24. Orthopantomogramme montrant une fracture spontanée de l'angle mandibulaire droit survenu dans le décours d'un traitement aux biphosphonates pour un myélome multiple (multiples lacunes bien visibles au niveau angulaire gauche).

\section{Tumeurs ou malformations vasculaires} intra-osseuses

Heureusement très rare, une telle lésion peut être mortelle par hémorragie intarissable, si elle est méconnue et qu'un banal geste chirurgical au sens large ne vienne à rompre une de ses frontières [28]. Toute biopsie, extraction ou la moindre agression chirurgicale risquent donc d'entraîner une hémorragie et comme à ce moment-là, souvent l'accès à la lésion est réduit, les moyens classiques de contrer cette hémorragie s'avèrent le plus souvent inefficaces. Les symptômes ne sont pas spécifiques : mobilité dentaire avec déplacement (la dent s'enfonce comme dans un coussinet), perception pulsatile gingivale ou osseuse, gingivorragies, signe de Vincent parfois. Radiologiquement, on peut percevoir soit une érosion osseuse de la corticale, des résorptions radiculaires, des troubles d'éruption dentaire, une image en bulles de savon ou rayons de soleil (images de cavités aréolaires avec zone de décalcification séparée par de fines cloisons, un élargissement apparent du canal mandibulaire dont les corticales sont érodées (Figures 25, 26). Le diagnostic nécessite une angiographie (ou un angioscan) (Figure 27) qui permet aussi le cas échéant, une embolisation thérapeutique [29]. Parfois, un curetage sera possible après embolisation, parfois une résection osseuse et greffe seront nécessaires. La radiothérapie est formellement contre-indiquée (risque élevé de sarcomatisation). 


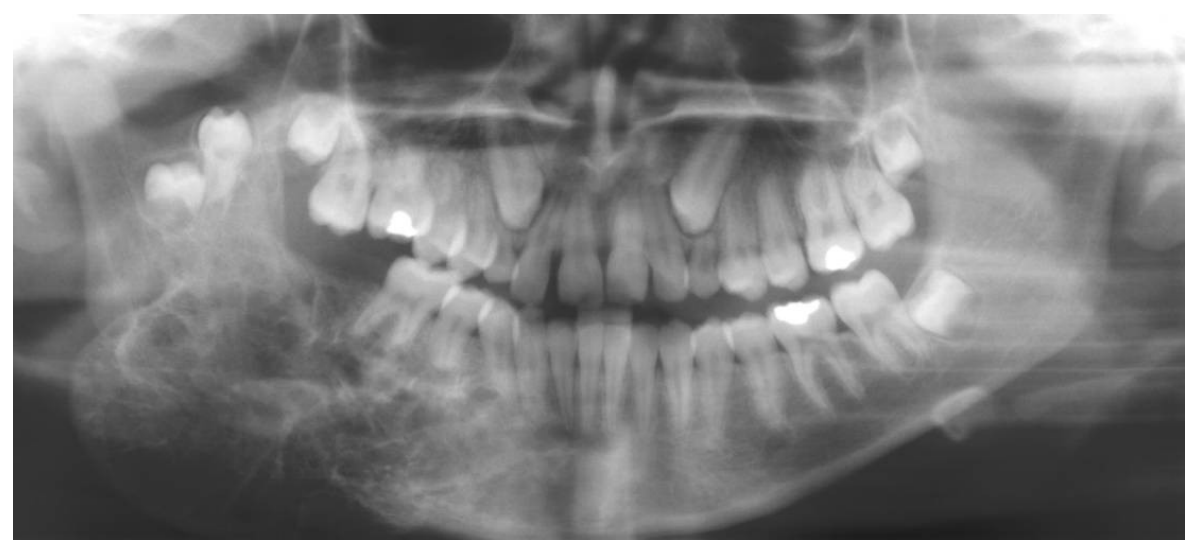

Fig. 25. Orthopantomogramme d'une malformation artério-veineuse de la branche horizontale droite de la mandibule

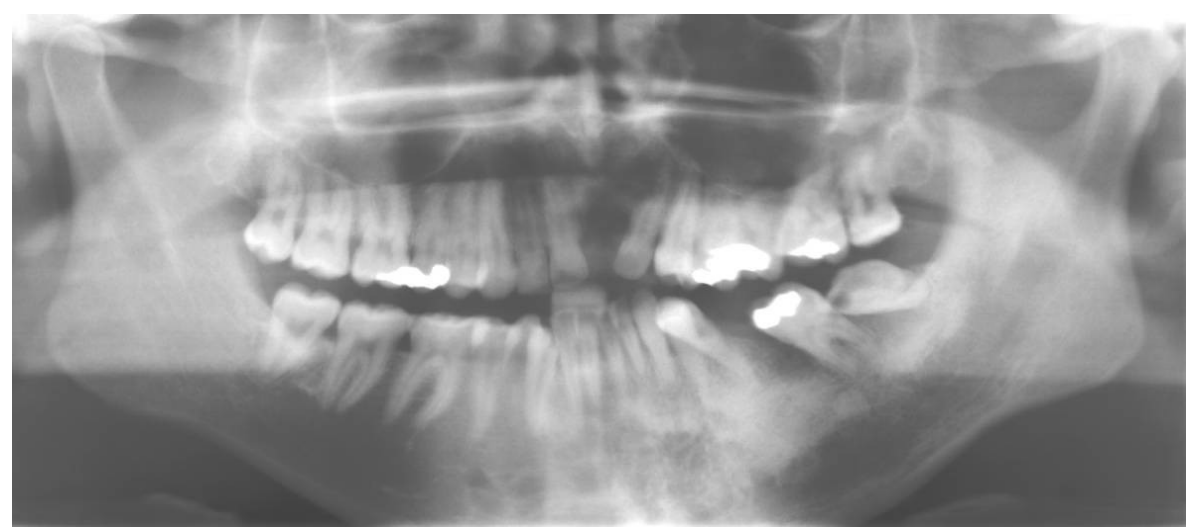

Fig. 26. Orthopantomogramme d'une malformation artério-veineuse de la branche horizontale gauche de la mandibule : élargissement du canal mandibulaire, disparition des corticales, signe de Vincent, multiples « logettes » radioclaires. 


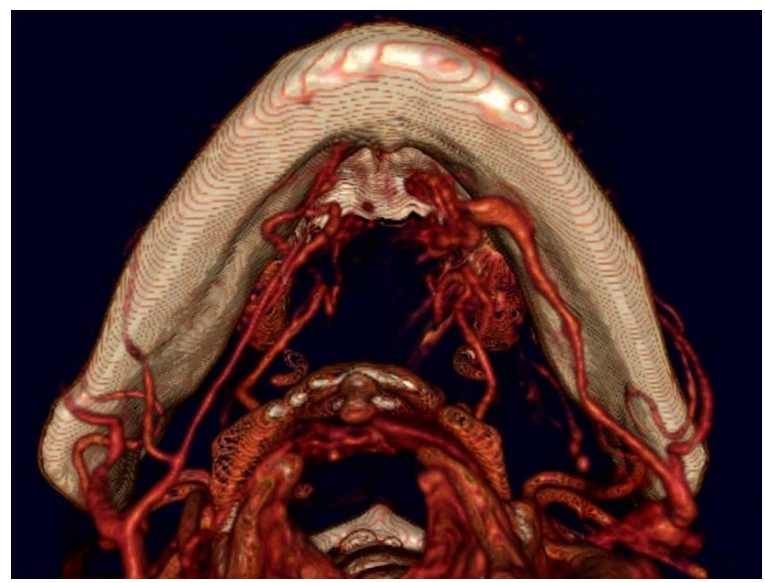

Fig. 27. Angioscan illustrant la visualisation d'axes vasculaires périmandibulaires.

\section{Tumeurs primitives osseuses}

Les tumeurs primitives à point de départ du tissu osseux sont des fibrosarcomes, des ostéosarcomes (Figure 28), des chondrosarcomes; celles à point de départ des tissus hématopoïétiques sont des réticulosarcomes, des plasmocytomes ou myélomes multiple. Elles sont toutes très rares et de très mauvais pronostic [30]. Les facteurs étiologiques sont mal connus et de plus en plus, les hypothèses génétiques sont retenues. Pour l'ostéosarcome, il peut survenir sur un traumatisme, après radiothérapie, sur une maladie de Paget [31] et sur toute tumeur bénigne osseuse. Leurs métastases hématogènes sont fréquentes et précoces, principalement dans le poumon, le foie ou les autres structures osseuses; la dissémination lymphatique et ganglionnaire, est exceptionnelle. Malheureusement, elles touchent plutôt les jeunes [32]. Rarement le diagnostic peut être clinique; un signe pathognomonique précoce pour l'ostéosarcome est l'élargissement de l'espace desmodontal, asymétrique (se rencontre sinon uniquement dans la sclérodermie) et l'élargissement du canal mandibulaire (rencontré aussi dans le neurofibrome intra-osseux, dans l'angiome mandibulaire et dans le lymphome non-hodgkinien) [33]; c'est l'examen anatomopathologique qui donnera le diagnostic définitif. L'aspect macroscopique est celui de chair à poisson ou de tissu encéphaloïde. Radiologiquement, on peut trouver des "flammèches", spicules osseux qui signent la réaction périostée ostéophytique, appelée image en feu d'herbe qui est typique de l'ostéosarcome; plus souvent, on constate la coexistence de zones ostéolytiques et ostéocondensantes. Le traitement est multidisciplinaire et fonction 
de nombreux facteurs [34]: âge du patient, type histologique de la tumeur, localisation tumorale, pronostic. Certaines tumeurs osseuses sont radiosensibles, d'autres sont chimiosensibles. Les résections osseuses peuvent être de plus en plus étendues grâce aux possibilités de reconstruction (par plaques vissées, par greffes osseuses libres), mais il faudra toujours mettre en balance les modifications postthérapeutiques (aspects esthétiques mais aussi aspects fonctionnels) et l'espoir de survie du patient, et survie alors dans un minimum de confort et d'insertion sociale possible (Figure 29). La radiothérapie comporte malheureusement toujours un certain risque d'ostéoradionécrose. La chimiothérapie est administrée selon des schémas associant plusieurs drogues pour augmenter leur efficacité, leur tolérance et diminuer leur toxicité; la radiothérapie et la chimiothérapie sont souvent combinées actuellement et de plus, des thérapies ciblées par des anticorps monoclonaux de plus en plus souvent associées à une de ces autres modalités thérapeutiques. Un soutien moral et psychologique de ces malades est indispensable. Le plus souvent, la combinaison de plusieurs formes de traitement est nécessaire et cela exige une étroite collaboration au sein d'une équipe pluridisciplinaire.

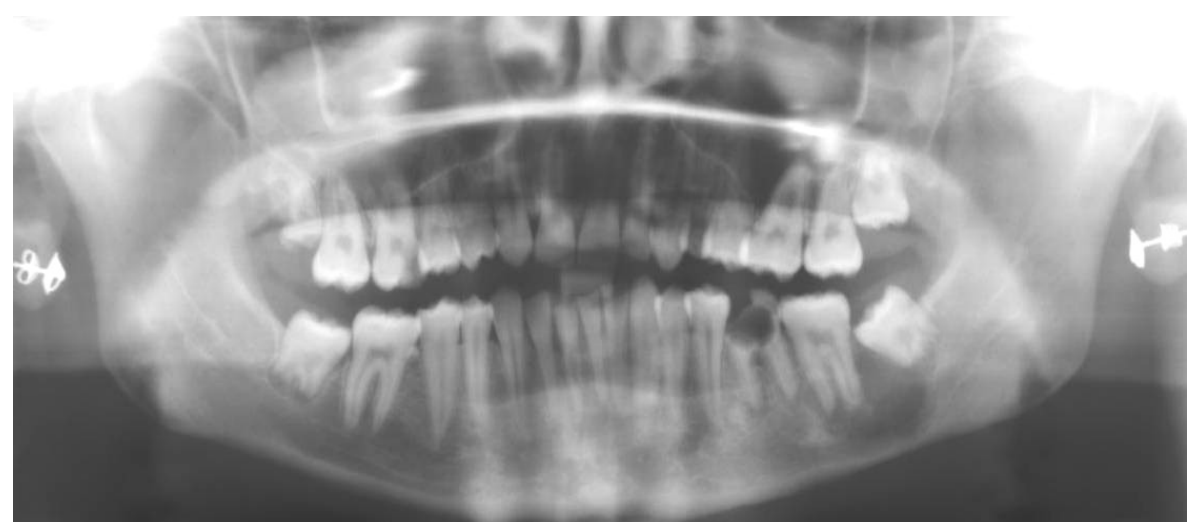

Fig. 28. Orthopantomogramme mettant en évidence une radio-clarté sous la dent $n^{\circ} 37$, mal délimitée, accompagnée d'une mobilité dentaire et d'un signe de Vincent, qui s'est révélée être un ostéosarcome. 


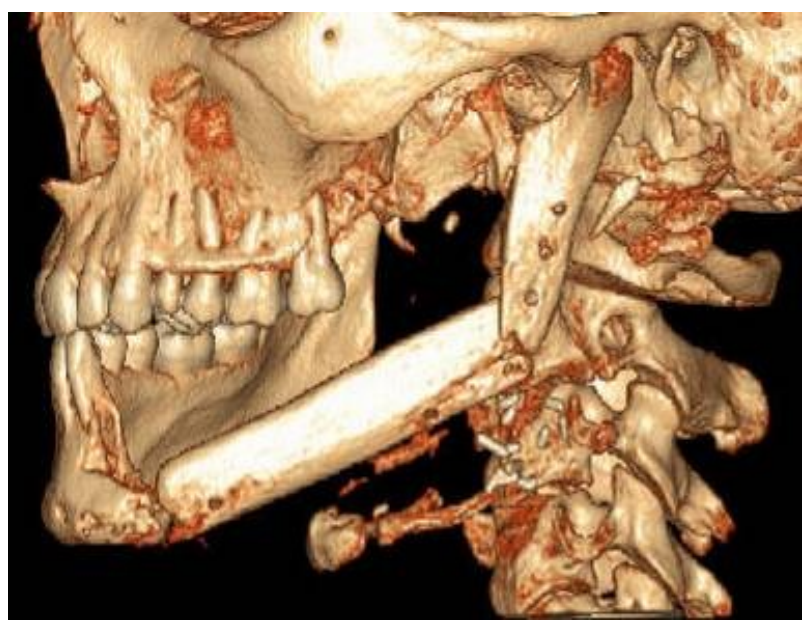

Fig. 29. CT scan tridimensionnel illustrant la résection et le résultat d'une reconstruction microchirurgicale par transfert de fibula.

Le plasmocytome (isolé ou multiple) et le myélome multiple (Figures 30, 31) résulte d'une prolifération de plasmocytes dans la moëlle osseuse qui produisent des immunoglobulines atypiques (chaînes légères); la tumeur touche préférentiellement l'homme âgé, au niveau de la région angulaire de la mandibule. Le plus souvent, plusieurs os sont atteints (crâne, maxillaires, côtes, colonne et bassin) [35]. La radiographie est assez typique, avec des lacunes lytiques comme à l'emporte-pièce. Le diagnostic sera histologique et basé sur la présence de protéines de Bence-Jones dans les urines (composant des Ig atypiques). Le diagnostic fait appel à différentes techniques d'imagerie, pour dresser le bilan d'extension et rechercher la tumeur primitive (le PET scan est actuellement l'examen de choix ). Le traitement est celui de la tumeur primitive; pour les métastases, il est le plus souvent palliatif (douleurs, pathologies locales). 


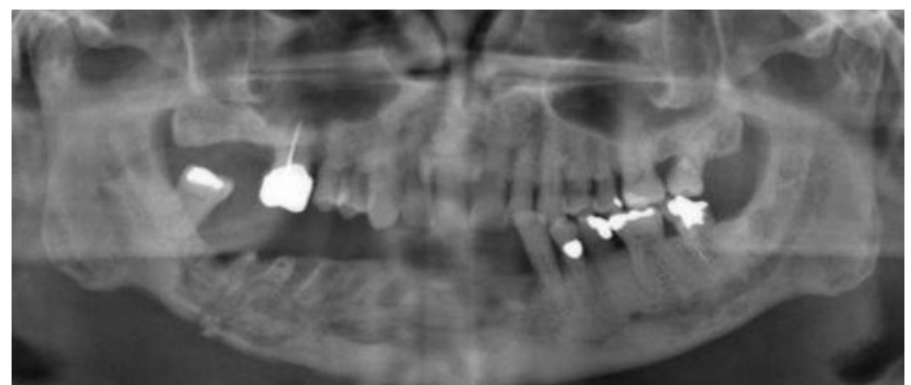

Fig. 30. Orthopantomogramme montrant une fracture spontanée de la mandibule dans un cas de myélome multiple (nombreuses lacunes intraosseuses, élargissement du canal bien visible du côté gauche).

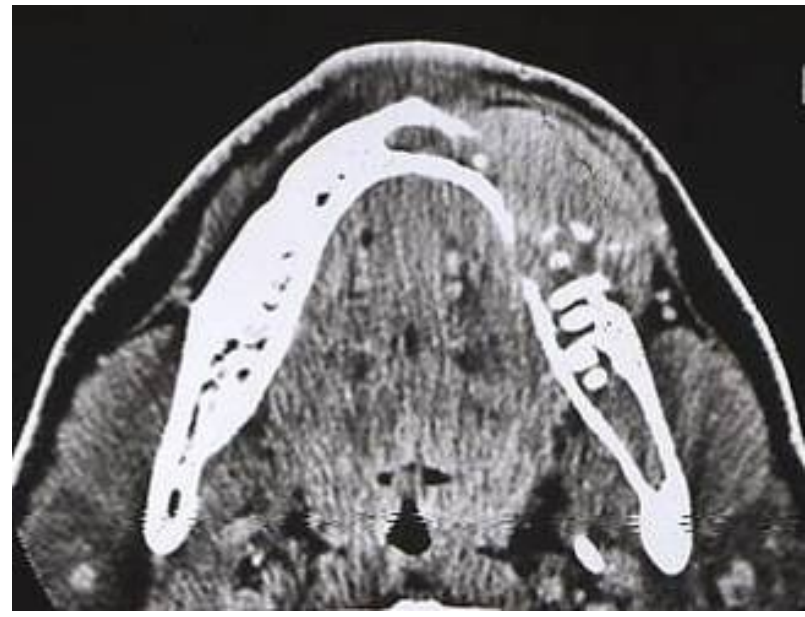

Fig. 31. CT scan axial mandibulaire d'un plamocytome isolé mandibulaire. ostéoclastome

Il s'agit probablement de l'équivalent intra-osseux du granulome giganto-cellulaire périphérique (épulis) : c'est une tumeur à malignité locale, touchant surtout les femmes jeunes, principalement à la mandibule. On considère que l'étiologie est une réponse cellulaire à un stimulus, peut-être vasculaire (infarcissement ?). Il n'y a ni 
douleurs, ni paresthésies; les dents sont bousculées ou égressées. Radiologiquement, on trouve des lacunes poly-lobulées, en images de bulles de savon; les corticales sont parfois détruites, les racines dentaires peuvent être résorbées (Figure 32). Le diagnostic différentiel doit être fait avec le granulome éosinophile, la dysplasie fibreuse, l'hyperparathyroïdie, le kyste anévrysmal, l'améloblastome. Le traitement est chirurgical : curetage mais des récidives sont fréquentes [36].

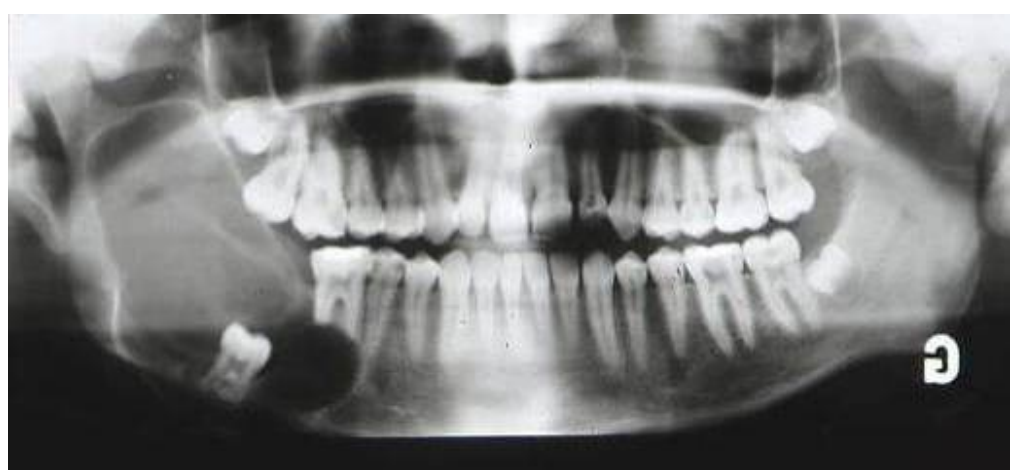

Fig. 32. Orthopantomogramme montrant un granulome giganto-cellulaire central de la mandibule droite : la dent $n^{\circ} 48$ manque, détruite par la tumeur, la dent $n^{\circ} 47$ est refoulée à la basilaire et la dent $n^{\circ} 46$ voit ses racines résorbées.

\section{Histiocytoses}

En 1987 la Histiocyte Society a introduit une nouvelle classification d'l'histiocytoses: classe I ou langerhansienne, classe II ou non-langerhansienne, et classe III ou histiocytose maligne. L'histiocytose langerhansienne est une lésion non néoplasique, granulomateuse (solitaire ou multifocale, avec ou sans atteinte des tissus mous), d'origine inconnue (réactionnelle?, auto-immune?) et le plus souvent présente chez les enfants de moins de 10 ans. Le diagnostic histologique est basé sur la présence d'une prolifération des cellules dendritiques, des histiocytes, des éosinophiles, des neutrophiles et des cellules plasmatiques. Pour rappel, les histiocytes sont des cellules présentant l'antigène aux lymphocytes $\mathrm{T}$ ou $\mathrm{B}$. Le diagnostic définitif est basé sur le marquage immunologique anti-CD1a [37]. Radiologiquement, il s'agit de radio-clarté mal délimitée, souvent uniloculaire. Des problèmes dentaires de mobilité, de résorptions radiculaires peuvent survenir. La progression des lésions s'effectue en épisodes espacés par des périodes de rémission plus ou moins longs. 
Des cas de guérison spontanée ont été rapportés [38]. Les présentations cliniques sont variables. Il existe trois formes d'histiocytose non-langerhansienne: la forme localisée ou granulome éosinophile, la forme multi-systémique chronique disséminée ou maladie de Hand-Schüller-Christian (lésions sont d'abord d'allure parodontales bénignes, puis osseuses, lytiques, diffuses; association à d'autres lésions cutanées, viscérales, neurologiques et au diabète insipide; radiologiquement, les dents paraissent suspendues dans l'os alvéolaire. Le traitement associe des mesures chirurgicales (curetage) à une chimiothérapie, à des corticoïdes) [39], et la forme multisystémique aigue disséminée ou maladie de Abt-Letterer-Siwe (souvent mortelle). Le pronostic de l'histiocytose langerhansienne est plus sombre s'il existe une lésion extra-osseuse, par exemple pulmonaire. Le traitement d'une lésion osseuse unique est chirurgical (curetage ou résection). La résection du périoste n'est pas recommandée. La radiothérapie est préconisée en cas de récidive locale ou si la chirurgie n'est pas possible. En cas de forme poly-ostotique la radiothérapie est le premier choix. La chimiothérapie est indiquée en cas de lésion est initialement systémique et multifocale. Le contrôle doit s'effectuer tous les trois mois, avec un suivi de 12-18 mois par imagerie médicale (CT scanner, scintigraphie) pour exclure ou diagnostiquer la progression de la lésion [40]. Il existe aussi une histiocytose non-langerhansienne dont la localisation osseuse est très rare. Cette pathologie bénigne, d'origine inconnue (réactionnelle?) affecte plutôt les tissus cutanés des garçons de moins de un an. L'involution spontanée est possible. Le diagnostic histologique est basé sur la prolifération de monocytes plasmocytoïdes. L'os peut être atteint dans les formes systémiques. Le traitement consiste en une exérèse-biopsie [41].

\section{Ankylose articulaire temporo-mandibulaire}

Pathologie grave car invalidante, l'ankylose temporo-mandibulaire doit être prévenue. Suite à un traumatisme, surtout méconnu ou maltraité, des noyaux de métaplasie osseuse apparaissent au sein d'un hématome intra-capsulaire, une synfibrose se transformant en synostose entre la base du crâne et le condyle mandibulaire. Parfois post-infectieuse (locale ou hématogène), plus rarement secondaire à des remaniements post-inflammatoires, l'ankylose articulaire se manifeste, en fonction de l'âge d'apparition de l'ankylose, par des troubles occlusaux, une asymétrie faciale, un profil d'oiseau, une atrophie musculaire et divers troubles fonctionnels plus ou moins invalidants. A terme, le retentissement psychosocial est tel qu'il justifie la lourdeur des traitements (Figure 33). Un traitement chirurgical de libération (avec ou sans interposition, p.ex. greffe chondro-costale), associé à une rééducation musculaire vise à lever la limitation d'ouverture buccale alors que des ostéotomies/greffes visent à corriger la dysmorphose maxillo-faciale secondaire. Des récidives de l'ankylose sont malheureusement très fréquentes, quel que soit le type de chirurgie libératrice utilisé [42]. 


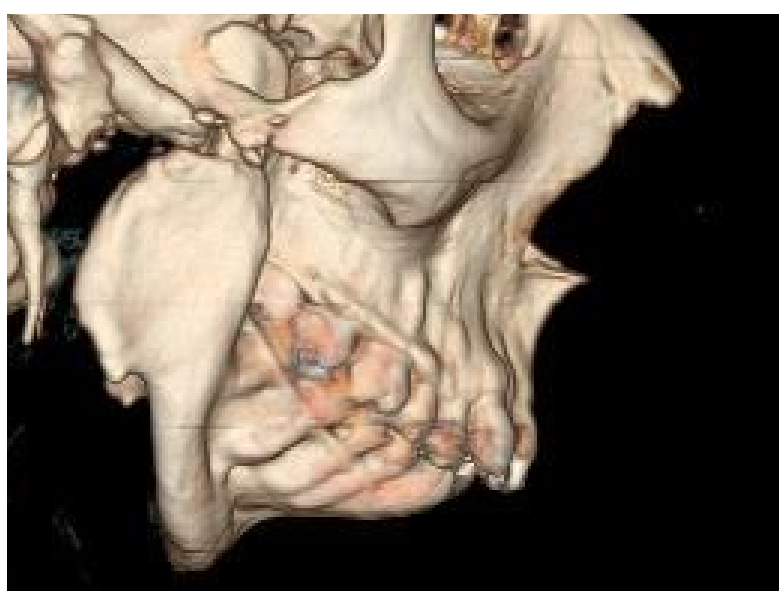

Fig. 33. CT scan 3D illustrant la déformation temporo-mandibulaire (ankylose) et la déformation mandibulaire inhérente aux troubles de croissance condylienne.

\section{Dysplasies osseuses fibrokystiques}

Il s'agit de maladies génétiques sporadiques (mutation du gène GNAS1 sur chromosome 20) caractérisées par une fibrose au sein de la moëlle osseuse avec simultanément une ostéolyse et une ostéogenèse.

La maladie de Jaffé-Lichtenstein existe sous deux formes, mono-ostotique (20 à 30 fois plus fréquente, pouvant être localisée aux maxillaires) et une forme polyostotique atteignant très rarement les maxillaires. C'est chez les enfants et les adolescents que l'affection est le plus souvent détectée. Suite à des stimuli inconnus survient une réaction osseuse désordonnée et disproportionnée.

Le chérubisme est une autre dysplasie fibrokystique bilatérale familiale autosomique dominante atteignant principalement les étages moyen et inférieur de la face des enfants, ce qui leur donne un aspect joufflu rappelant celui de chérubin. L'os est formé de tissu fibreux et ostéoïde [43].

La maladie de Mc Cune-Albright est une maladie caractérisée par des dysplasies fibreuses des maxillaires, des taches "café au lait" cutanées, une pathologie endocrinienne (p. ex. gonadique : puberté précoce) et une maturation précoce du squelette.

Les différentes variantes génétiques sont actuellement bien connues [44]. Les symptômes qui en résultent sont une asymétrie faciale par tuméfaction (Leontiasis ossea est un aspect léonin du visage), des dents déplacées ou incluses ou résorbées; les lésions peuvent éventuellement envahir les sinus de la face ou la base du crâne. Il 
n'y a pas de douleurs. Tous les tests biologiques -témoins du métabolisme osseuxsont normaux. Radiologiquement, on trouve un aspect floconneux de l'os, ouaté, pseudo-kystique avec des aires juxtaposées de raréfaction et de condensation, aux limites imprécises, sans contours nets, d'allure parfois pseudo-tumorale (Figures 3436). Le diagnostic différentiel est assez facilement fait avec la maladie de Paget, l'hyperparathyroïdie, l'ostéofibrome ou le fibrome ossifiant juvénile. L'évolution se fait soit par régression spontanée après maturation du squelette, soit par maturation en ostéome soit heureusement rarement en sarcomatisation. Le traitement des dysplasies fibreuses consiste le plus souvent en une surveillance; un curetage chirurgical modelant peut être réalisé à la demande (pour raison esthétique par exemple) mais jamais très tôt, car cela risque toujours d'induire une maturation du squelette et des troubles de croissance. Des ostéotomies des maxillaires sont cependant possibles si indispensables [44].

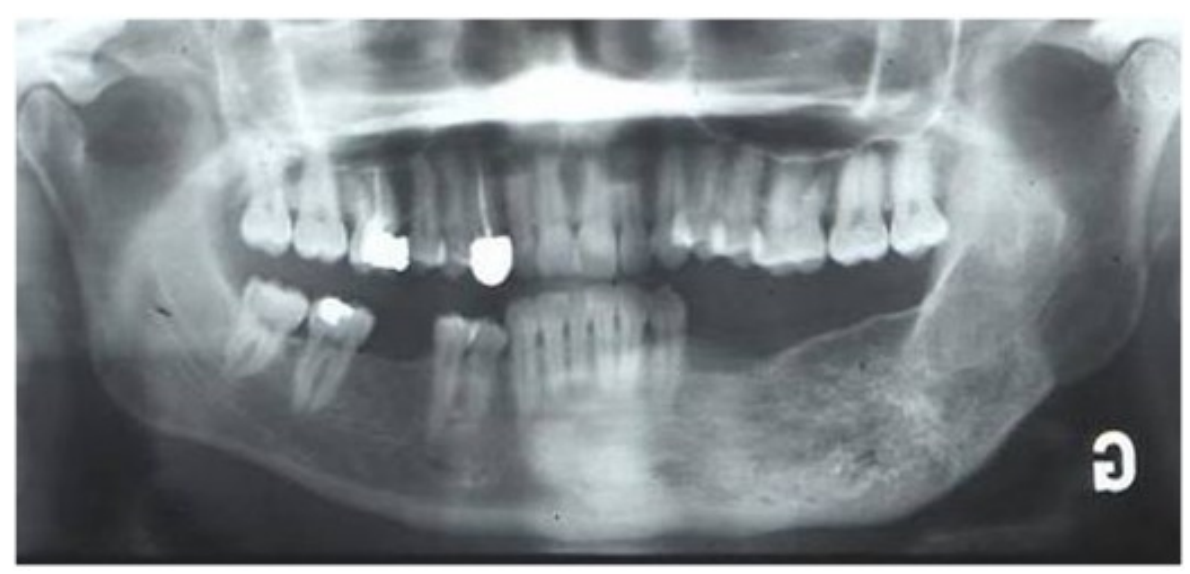

Fig. 34. Orthopantomogramme illustrant une dysplasie fibreuse de la branche horizontale gauche de la mandibule. 


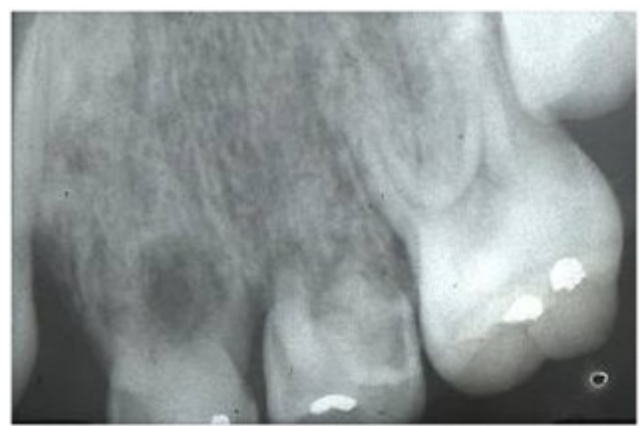

Fig. 35. Radiographie rétro-alvéolaire illustrant des images typiques de dysplasie fibreuse.

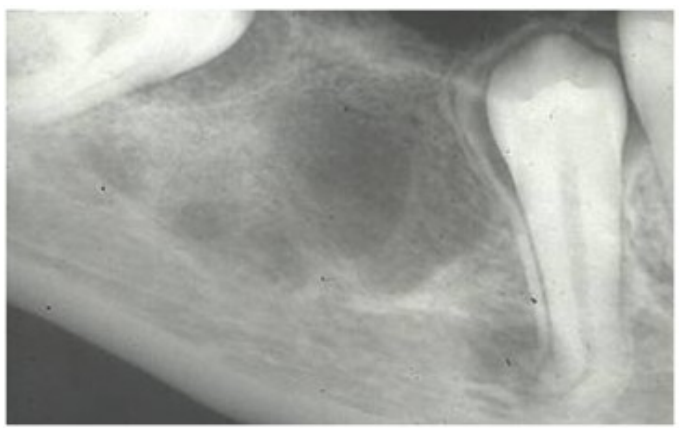

Fig. 36. Extrait d'un orthopantomogramme illustrant des images typiques de dysplasie fibreuse.

\section{Ostéomyélite chronique primaire}

L'ostéomyélite chronique primaire est une entité nosologique particulière rapportée sous de nombreux synonymes : ostéomyélite sclérosante diffuse, ostéomyélite ossifiante, ostéomyélite sèche, ostéomyélite chronique sclérosante non suppurative, ou hyperplasie osseuse réactionnelle. Par définition, elle n'est donc pas secondaire à une infection primaire non ou mal traitée [45]. L'étiologie est controversée : origine bactérienne (Actinomycètes ? Eikenella corrodens?), virale (herpès?), toxinique, thrombotique veineuse inflammatoire. L'hypoxie qui en résulte favoriserait la colonisation osseuse par des bactéries tels les Propionibacterium acnes et les Peptos- 
treptococcus intermedius. L'évolution chronique de la maladie s'expliquerait par un défaut immunitaire local. Son incidence est en augmentation constante. L'affection survient à tout âge mais le plus souvent chez les jeunes adultes (âge moyen de 27 ans); le sexe féminin est 4 fois plus atteint. La localisation est exclusivement mandibulaire, plus particulièrement la région angulaire, avec extension exceptionnelle aux structures articulaires temporo-mandibulaires et osseuses temporales et zygomatiques. Le côté droit serait le plus fréquemment atteint chez les femmes tandis que ce serait le côté gauche chez les hommes. D'autres os (extra-faciaux) peuvent être beaucoup plus rarement atteints. Des épisodes douloureux, d'une durée de une à deux semaines, se répètent à intervalles plus ou moins distants de un à plusieurs mois, mais se reproduisent de moins en moins fréquemment au fur et à mesure que l'évolution se poursuit vers la sclérose. Le bord inférieur de la mandibule et la région angulo-mandibulaire s'hypertrophient par suite d'une réaction périostée, au point d'entraîner une asymétrie du tiers inférieur de la face. Un trismus, d'abord intermittent puis permanent, accompagne les épisodes douloureux; une réelle ankylose temporo-mandibulaire peut s'installer. Il ne survient jamais de fistulisation. Il n'y a aucun symptôme ni dentaire ni parodontal; la muqueuse orale peut être érythémateuse et œdématiée, mais uniquement pendant les accès aigus. Une adénopathie cervicale d'aspect inflammatoire est peu fréquente. Les signes généraux comme de la fièvre, sont toujours réduits. Les signes radiologiques sont assez évocateurs quoique variables avec l'âge du sujet et des lésions. A un stade précoce, une réaction périostée peut être présente. Des zones d'ostéolyse et de sclérose osseuse ne sont perceptibles qu'après une évolution d'au moins un mois. A ce stade, le diagnostic différentiel radiologique est difficile avec les autres formes d'ostéomyélite et les tumeurs malignes intra-osseuses, et dans ces cas seule l'anatomo-pathologie permettra le diagnostic. Une perte de substance osseuse corticale localisée à l'angle mandibulaire est classique. Après quelque temps d'évolution chez l'enfant, l'asymétrie mandibulaire apparaît par épaississement osseux aussi bien en hauteur qu'en largeur. Plus tard ou chez l'adulte, le volume mandibulaire tend à se normaliser sauf au niveau des coroné et condyle mandibulaires qui restent épaissis. Les racines dentaires sont résorbées. Les zones ostéolytiques apparaissent souvent en même temps que ne surviennent les épisodes douloureux, puis régressent pour être progressivement remplacées par des zones ostéosclérotiques, homogènes et sans délimitation nette par rapport à l'os avoisinant. Les radiographies conventionnelles n'ont qu'un faible interêt diagnostique (Figure 37). La scintigraphie osseuse met les zones ostéolytiques mieux et plus précocement en évidence que les clichés radiographiques, et est utile à la surveillance de l'évolution. La tomodensitométrie est utile au diagnostic mais très peu spécifique. L'IRM est supérieure à la scintigraphie dans l'appréciation de l'extension des processus infectieux au sein des tissus mous. Le diagnostic radiologique différentiel doit être fait avec les lésions mono-ostotiques de dysplasie fibreuse (indolores, localisées au maxillaire supérieur, ayant l'aspect radiologiquement classique en pelures 
d'orange, sans réaction périostée ni résorption radiculaire des dents), la maladie de Paget (plurifocale, atteignant rarement les jeunes, hypercémentose, augmentation des phosphatases alcalines sériques et certaines particularités histologiques), la périostite de Garré (réaction périostée en regard d'une dent causale infectée, peu douloureuse), le cémentome (asymptomatique, plurifocal mais toujours limité à la région alvéolaire, sans réaction périostée, découverte histologique de cément) et des tumeurs malignes comme les sarcomes, les lymphomes non hodgkiniens et les métastases osseuses. L'anatomo-pathologie n'est nullement spécifique : ostéosclérose, apposition osseuse périostée et cellules inflammatoires. Le diagnostic fait appel aux éléments cliniques, radiologiques et histologiques. La mise en évidence bactériologique du germe étiologique est toujours difficile et requiert une technique rigoureusement aseptique; elle permet toutefois de diriger le traitement antibiotique. Le traitement, décevant car inefficace quant à l'éradication radicale du processus, comporte un volet médical et un volet chirurgical. Les antibiotiques sont utiles au début de l'évolution et prescrits durant plusieurs mois de manière dirigée si possible. Les corticoïdes, prescrits en l'absence de germe étiologique pendant maximum 10 jours et plutôt lors des accès douloureux des récidives, sont utiles comme agents antiinflammatoires. Les anti-inflammatoires non stéroïdiens sont peu efficaces. Le traitement par les biphosphonates a été récemment préconisé. L'oxygénothérapie hyperbare est actuellement préconisée en raison de son efficacité sur les germes anaérobies et de la stimulation de l'angiogenèse, permettant à la fois un meilleur apport antibiotique au sein des lésions osseuses et une cicatrisation. Le traitement chirurgical de choix est la décortication et l'élimination des foyers nécrotiques et séquestrés, indiquées en cas d'échec des traitements médicaux et en cas d'évolution continue de l'affection; elles peuvent être combinées à une apposition de greffons osseux spongieux autologues, à une irrigation permanente de solutions contenant des antibiotiques, des antiseptiques, ou encore des substances protéolytiques, ou encore à la mise en place de billes de résine imprégnées de gentamicine ou de tobramycine. En cas de processus infectieux très étendus, une exérèse plus large peut être indiquée. Les extractions dentaires en zone ostéomyélitique doivent être faites sous le couvert d'une antibiothérapie [46]. 


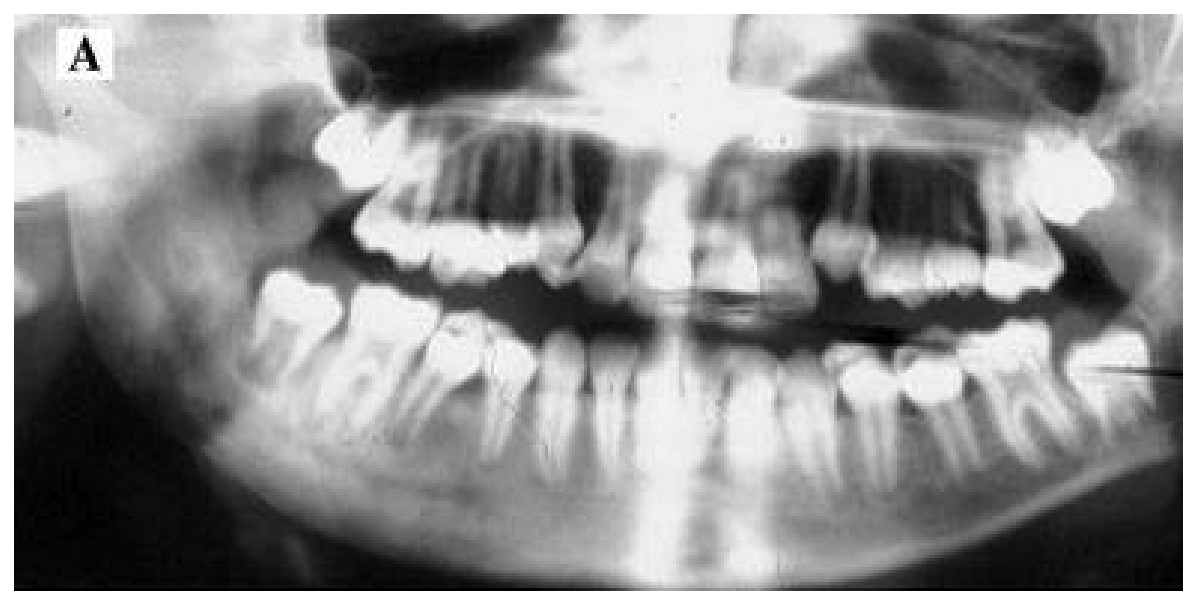

Fig. 37. Orthopantomogramme illustrant la sclérose osseuse mandibulaire droite, la disparition des corticales du canal mandibulaire ainsi que du desmodonte des molaires et prémolaires inférieures droites : ostéomyélite chronique primaire.

\section{Ostéite actinomycosique}

Elle est caractérisée par un mode d'évolution chronique due à une infection, ostéite par Actinomyces israeli, qui a pu pénétrer au sein de l'os au travers d'une lésion carieuse dentaire, d'une lésion muqueuse ou d'une plaie d'extraction. Le maxillaire supérieur est aussi souvent atteint que la mandibule. Les signes radiologiques sont aspécifiques et constitués d'une ostéolyse diffuse, rarement térébrante ni séquestrante (Figure 38). Le traitement est médical et chirurgical : une antibiothérapie dirigée et de longue durée, en raison de la pathogénie de ces germes entourés d'une coque de phospholipides, doit être accompagnée d'un curetage et d'une élimination des séquestres osseux. Les très fréquentes récidives ou pseudo-résistances à ces traitements s'expliquent par la méconnaissance de l'étiopathogénie et des caractéristiques microbiologiques de ces germes [47]. 


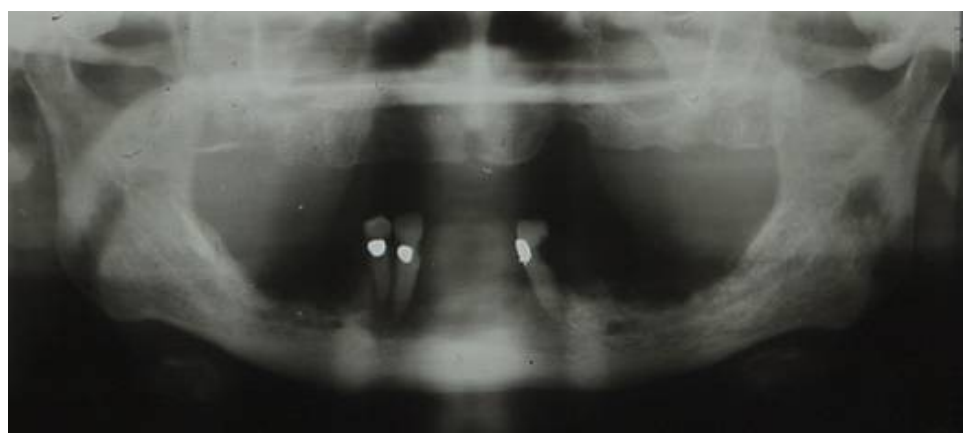

Fig. 38. Orthopantomogramme montrant une ostéite actinomycosique des deux remparts alvéolaires inférieurs.

\section{Sinusite maxillaire chronique}

La sinusite maxillaire chronique d'origine dentaire est beaucoup plus fréquente que la sinusite aiguë [48]. Le plus souvent, elle est unilatérale et d'installation progressive. Divers mécanismes étiopathogéniques doivent être distingués. En cas de périodontite chronique périapicale, les phénomènes inflammatoires et infectieux périapicaux peuvent engendrer une réaction hyperplasique avec inflammation chronique de la muqueuse antrale, d'abord en regard de la lésion dentaire, ensuite de manière diffuse dans tout le sinus maxillaire, voire plus exceptionnellement sous forme d'une pan-sinusite. Dans $5 \%$ des cas, un corps étranger introduit accidentellement au niveau du sinus maxillaire (une dent incluse, la dent de sagesse supérieure le plus souvent, parfois une prémolaire, rarement la canine, un implant dentaire, un débris radiculaire ou un matériau d'obturation endodontique ayant dépassé l'apex) peut occasionner une sinusite chronique. Ces corps "étrangers" sont responsables d'irritation mécanique, chimique et bactérienne, donnant lieu secondairement à la réaction hyperplasique inflammatoire sinusale. Les parodontopathies ne sont qu'exceptionnellement responsables de sinusite maxillaire chronique [48]. Cliniquement, les douleurs sont peu importantes voire inexistantes. Des céphalées frontales ou orbitaires peuvent survenir. Une tuméfaction jugale ou sous-orbitaire et un trouble de la sensibilité dans le territoire de la branche maxillaire du nerf trijumeau plaide le plus souvent en faveur de la présence d'une tumeur maligne. Les adénopathies régionales et des signes généraux infectieux sont exceptionnels. Les dents en cause ne sont que rarement douloureuses à la percussion et à la pression. La recherche d'une étiologie dentaire doit cependant rester systématique. Une fistule ou 
communication oro-sinusale doit être minutieusement recherchée. La rhinoscopie montre souvent des sécrétions muco-purulentes dans la fosse nasale, issues du méat moyen et permet de suivre les sécrétions purulentes [48].

C'est essentiellement l'imagerie médicale actuellement de manière standard par un CT scan, qui objective les lésions [48]: opacification homogène, toujours à comparer avec le côté sain, intégrité des diverses parois osseuses, existence d'une image de niveau hydro-aérique, image en «cadre» (Figure 39), résultant de l'épaississement muqueux sinusal ou une opacité circonscrite au plancher sinusal. La radiographie panoramique ne donne qu'une piètre image de l'opacité sinusale mais garde sa valeur de dépistage des pathologies dentaires [48]. La résonance magnétique nucléaire n'a d'intérêt qu'en cas de diagnostic différentiel nécessaire avec une pathologie tumorale. Le diagnostic différentiel clinique et radiologique, qui n'est pas aisé, doit prendre en considération les sinusites maxillaires d'origine nasale, l'aéro-sinusite (liée aux modifications de pression atmosphérique lors de vols en haute altitude par exemple), l'aspergillome sinusal maxillaire, et les processus tumoraux bénins et malins du sinus maxillaire. Le traitement a pour but de restituer la fonction mucociliaire sinusale, de permettre l'aération du sinus maxillaire et, si nécessaire, d'enlever chirurgicalement la cause de la sinusite. Le traitement médical est constitué d'inhalations par voie nasale de décongestionnants et de mucolytiques; une sinusomanométrie permet d'apprécier l'état fonctionnel de l'ostium. Une antibiothérapie par voie orale sera administrée en cas de signes infectieux locaux et généraux importants. En cas de persistance des symptômes malgré une antibiothérapie bien conduite, le rinçage du sinus peut être pratiqué, éventuellement au travers de la fistule s'il y en a une. Le traitement chirurgical se fait en dernier recours, par méatotomie. La plupart des corps étrangers, pour autant que leur taille le permette, peuvent être retirés par sinusoscopie.

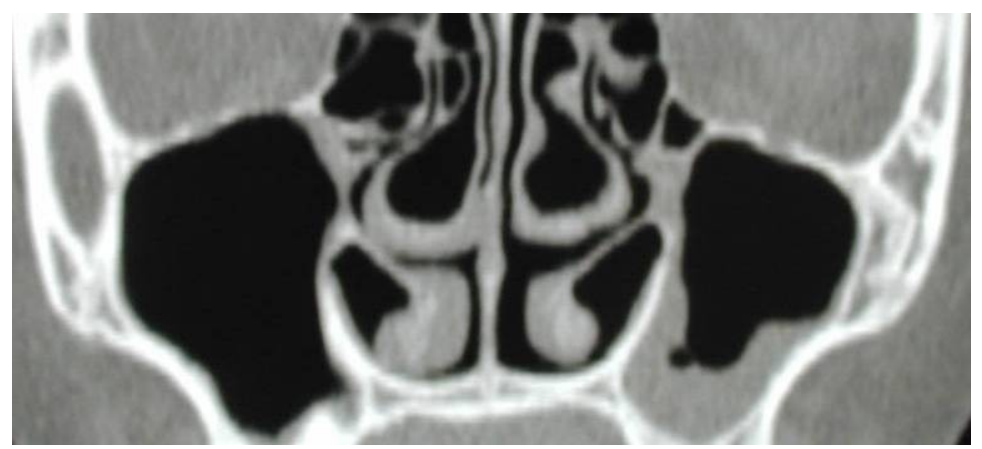

Fig. 39. CT scan coronal illustrant une sinusite chronique du sinus maxillaire gauche avec un épaississement dans le récessus alvéolaire. 


\section{Aspergillome sinusal}

L'aspergillose du sinus maxillaire est une mycose due à un champignon cosmopolite et ubiquitaire, du genre Aspergillus, classe des Hyphomycètes, famille des Moniliacées [49]. Il en existe environ 300 espèces, dont un grand nombre sont des saprophytes dans la nature. Chez l'Homme, ce sont surtout les voies aéro-digestives, la peau, la cornée, le conduit auditif externe et les voies urogénitales qui sont de sites primaires d'infections par Aspergillus. Les aspergilloses regroupent des syndromes cliniques où sont retrouvées une sensibilisation, une colonisation mycotique ou une invasion tissulaire par les champignons. On distingue ainsi une aspergillose allergique (trachéo-bronchique principalement), l'aspergillome isolé, par exemple sinusal, et l'aspergillose disséminée, principalement rencontrée chez des sujets immunodéprimés. Aspergillus fumigatus est l'agent principal responsable des sinusites mycotiques [50]. Plus fréquente chez l'homme que chez la femme, l'aspergillome du sinus maxillaire survient chez l'adulte présentant des conditions locales favorisantes : mauvaise aération sinusale, corps étranger intra-sinusal (surtout matériau d'obturation dentaire [51], polypose) (Figures 40, 41). Il a été démontré qu'un environnement anaérobique intra-sinusal et de faibles concentrations d'oxygène diffusant au départ des pâtes d'obturation dentaire à base de zinc, constituaient des facteurs favorisant le développement des aspergillus [52]

L'atteinte est le plus souvent unilatérale : obstruction nasale et mouchage purulent et/ou sanguinolent, parfois cacosmie, douleurs faciales, céphalées unilatérales et parfois crises d'éternuements. Les dents adjacentes peuvent être mobiles. En cas de forme agressive (immunodépression), la joue peut être fort gonflée, et une exophtalmie peut être retrouvée. Les signes radiologiques sont une opacité sinusale, les parois osseuses étant érodées ou détruites. Le diagnostic différentiel est celui des sinusites maxillaires. Le traitement est chirurgical, avec exérèse du remaniement muqueux et administration soit topique soit générale d'antimycotiques. 


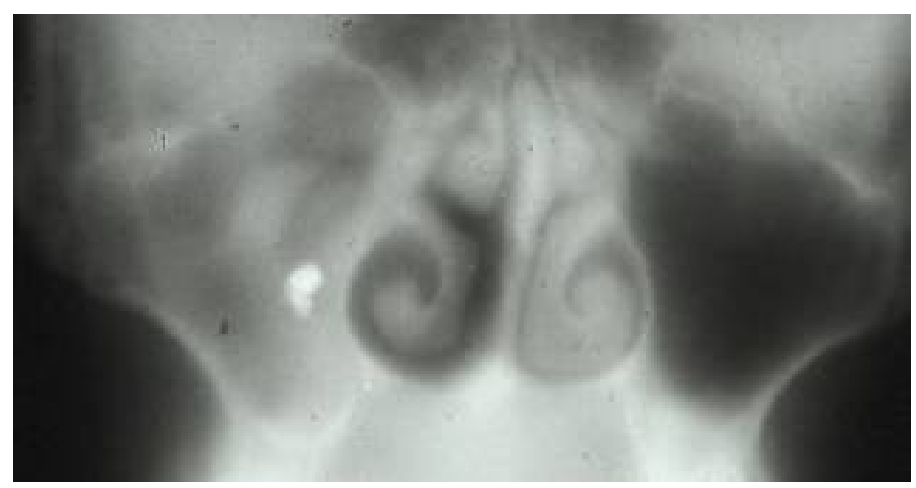

Fig. 40. Tomographie frontale montrant une opacité inhomogène du sinus maxillaire droit et un «corps étranger» très radio-opaque au centre du sinus, très suspect d'aspergillome.

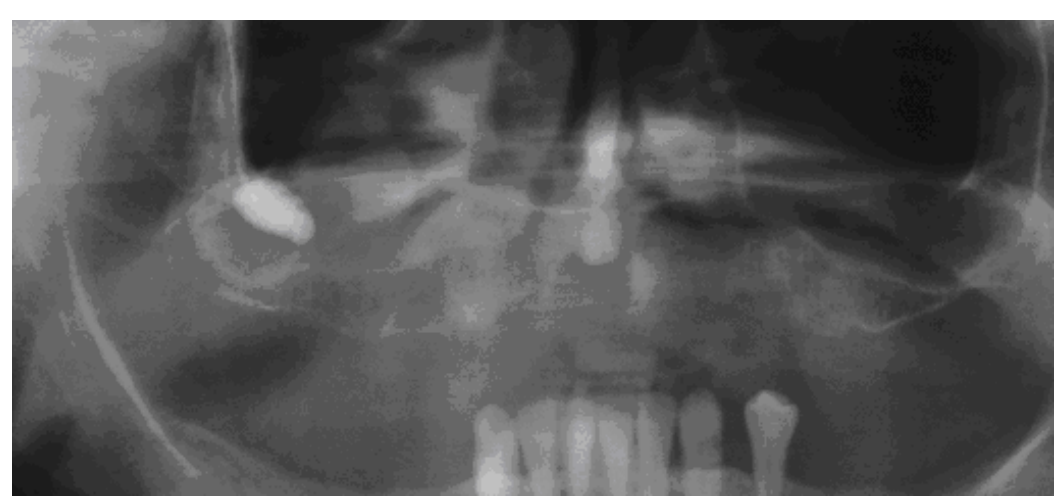

Fig. 41. Orthopantomogramme montrant une opacité inhomogène du sinus maxillaire droit et un «corps étranger» très radio-opaque au centre du sinus, très suspect d'aspergillome.

- Remerciements: aucun.

- Sources de financement: cette étude n'a reçu aucun soutien financier.

- Conflit d'intérêt: Pr Raphael Olszewski est l'Editeur-en-Chef de la revue NEMESIS. Pr Hervé Reychler déclare qu'il n'a pas de conflit d'intérêt lié à cette étude.

Conformité avec les standards éthiques 
- Approbation éthique: cette revue de la littérature n'a pas nécessité d'accord de comité d'éthique.

- Consentement éclairé: ce travail de revue de la littérature n'a pas nécessité de consentement éclairé de la part de patients. Toutes les images sont anonimisées.

\section{Contributions des auteurs}

\begin{tabular}{|l|l|}
\hline Auteur & Contribution \\
\hline Olszewski R & $\begin{array}{l}\text { Concept, Recherches, Méthodologie, Données, } \\
\text { Supervision, Validation, Visualisation, Préparation de } \\
\text { manuscrit, Ecriture-corrections et édition }\end{array}$ \\
\hline Reychler H & Supervision, Ecriture-corrections et édition \\
\hline
\end{tabular}

\section{Références}

1. Sharif FN, Oliver R, Sweet C, Sharif MO. Interventions for the treatment of keratocystic odontogenic tumours (KCOT, odontogenic keratocysts (OKC)). Cochrane Database Syst Rev 2010; 8: CD008464.

2. Lo Muzio L. Nevoid basal cell carcinoma syndrome (Gorlin syndrome). Orphanet J Rare Dis 2008;3:32-48. Review.

3. Kubota Y, Nitta S, Oka S, Nakagawa S, Ninomiya T, Shirasuna K. Discrimination of ameloblastomas from odontogenic keratocysts by cytokine levels and gelatinase species of the intracystic fluids. J Oral Pathol Med 2001; 30:421-427.

4. Saito T, Okada H, Akimoto Y, Yamamoto H. Primary intraosseous carcinoma arising from an odontogenic cyst: a case report and review of the Japanese cases. J Oral Sci 2002;44:49-53. Review.

5. Ide F, Mishima K, Yamada H, Horie N, Saito I, Shimoyama T, Kusama K. Unsuspected small ameloblastoma in the alveolar bone: a collaborative study of 14 cases with discussion of their cellular sources. J Oral Pathol Med 2008;37:221-227. Review.

6. Vanoven BJ, Parker NP, Petruzzelli GJ. Peripheral ameloblastoma of the maxilla: a case report and literature review. Am J Otolaryngol 2008;29:357-360. Review. 
7. Pogrel MA, Montes DM. Is there a role for enucleation in the management of ameloblastoma? Int J Oral Maxillofac Surg 2009;38:807-812.

8. Bridle C, Visram K, Piper K, Ali N. Maxillary calcifying epithelial odontogenic (Pindborg) tumor presenting with abnormal eye signs: case report and literature review. Oral Surg Oral Med Oral Pathol Oral Radiol Endod 2006;102:e12-15. Review.

9. Ching AS, Pak MW, Kew J, Metreweli C. CT and MR imaging appearances of an extraosseous calcifying epithelial odontogenic tumor (Pindborg tumor). AJNR Am J Neuroradiol 2000; 21:343-345. Review.

10. De Riu G, Meloni SM, Contini M, Tullio A. Ameloblastic fibro-odontoma. Case report and review of the literature. J Craniomaxillofac Surg 2010;38:141144. Review.

11. Bregni RC,Taylor AM, Garcia AM. Ameloblastic fibrosarcoma of the mandible: report of two cases and review of the literature. J Oral Pathol Med 2001;30:316-320.

12. Kobayashi K, Murakami R, Fujii T, Hirano A. Malignant transformation of ameloblastic fibroma to ameloblastic fibrosarcoma: case report and review of the literature. J Craniomaxillofac Surg 2005;33:352-355. Review.

13. Leiser Y, Abu-El-Naaj I, Peled M. Odontogenic myxoma--a case series and review of the surgical management. J Craniomaxillofac Surg 2009; 37:206-209. Review.

14. Kaffe I, Naor H, Buchner A. Clinicat and radiological features of odontogenic myxoma of the jaws. Dentomaxillofac Radiol 1997;26:299-303. Review.

15. Lugakingira M, Pytynia K, Kolokythas A, Miloro M. Primary intraosseous carcinoma of the mandible: case report and review of the literature. J Oral Maxillofac Surg 2010;68:2623-2629. Review.

16. Lin $\mathrm{YJ}$, Chen $\mathrm{CH}$, Wang WC, Chen YK, Lin LM. Primary intraosseous carcinoma of the mandible. Dentomaxillofac Radiol 2005;34:112-116.

17. MacDonald-Jankowski DS. Ossifying fibroma: a systematic review. Dentomaxillofac Radiol 2009;38:495-513. Review.

18. Liu Y, Wang H, You M, Yang Z, Miao J, Shimizutani K, Koseki T. Ossifying fibromas of the jaw bone: 20 cases. Dentomaxillofac Radiol 2010;39:57-63. Review. 
19. Nonaka CF, Pacheco DF, Nunes RP, Freitas Rde A, Miguel MC. Ossifying fibromyxoid tumor in the mandibular gingiva: case report and review of the literature. J Periodontol 2009; 80:687-692. Review.

20. Su L, Weathers DR, Waldron CA. Distinguishing features of focal cementoosseous dysplasias and cemento-ossifying fibromas: I. A pathologic spectrum of 316 cases. Oral Surg Oral Med Oral Pathol Oral. Radiol Endod 1997;84:301309.

21. Sun ZJ, Sun HL, Yang RL, Zwahlen RA, Zhao YF. Aneurysmal bone cysts of the jaws. Int J Surg Pathol 2009;17:311-322. Review.

22. Trent C, Byl FM. Aneurysmal bone cyst of the mandible. Ann Otol Rhinol Laryngol 1993;102:917-924. Review.

23. Chrcanovic BR, Reher P, Sousa AA, Harris M. Osteoradionecrosis of the jaws-a current overview--part 1: Physiopathology and risk and predisposing factors. Oral Maxillofac Surg 2010;14:3-16. Review.

24. Pitak-Arnnop P, Sader R, Dhanuthai K, Masaratana P, Bertolus C, Chaine A, Bertrand JC, Hemprich A. Management of osteoradionecrosis of the jaws: an analysis of evidence. Eur J Surg Oncol 2008;34:1123-1134. Review.

25. Gevorgyan A, Enepekides DJ. Bisphosphonate-induced necrosis of the jaws: a reconstructive nightmare. Curr Opin Otolaryngol Head Neck Surg 2008;16:325330. Review.

26. Devogelaer JP, Reginster JY, Rompen E, Boutsen Y, Body JJ, Bergmann P, Rozenberg S, Gaspard U, Hérion F, Reychler H. L'ostéonécrose maxillaire associée aux traitements aux biphosphonates dans le traitement de l'ostéoporose. Consensus interdisciplinaire. Louvain Med 2010;305-309.

27. Filleul O, Crompot E, Saussez S. Bisphosphonate-induced osteonecrosis of the jaw: a review of 2,400 patient cases. J Cancer Res Clin Oncol 2010;136:11171124. Review.

28. Collin AC, Viremouneix L, Guibaud L, Breton P. Intraosseous arteriovenous malformations. Rev Stomatol Chir Maxillofac 2010;111:11-18. Review.

29. Kacker A, Heier L, Jones J. Large intraosseous arteriovenous malformation of the maxilla - a case report with review of literature. Int $\mathrm{J}$ Pediatr Otorhinolaryngol 2000;52:89-92. Review.

30. Yamaguchi S, Nagasawa H, Suzuki T, Fujii E, Iwaki H, Takagi M, Amagasa T. Sarcomas of the oral and maxillofacial region: a review of 32 cases in 25 years. Clin Oral Investig 2004;8:52-55. 
31. Cheng YS, Wright JM, Walstad WR, Finn MD. Osteosarcoma arising in Paget's disease of the mandible. Oral Oncol 2002;38:785-792. Review.

32. Gadwal SR, Gannon FH, Fanburg-Smith JC, Becoskie EM, Thompson LD. Primary osteosarcoma of the head and neck in pediatric patients: a clinicopathologic study of 22 cases with a review of the literature. Cancer 2001;91:598-605. Review.

33. Buric N, Jovanovic G, Radovanovic Z, Buric M, Tijanic M. Radiographic enlargement of mandibular canal as first feature of non-Hodgkin's lymphoma. Dentomaxillofac Radiol 2010;39:383-388.

34. Brady JS, Chung SY, Marchiano E, Eloy JA, Baredes S, Park RCW. Pediatric head and neck bone sarcomas: An analysis of 204 cases. Int J Pediatr Otorhinolaryngol. 2017;100:71-76.

35. Bencheikh R, Benhammou A, Rabeh G, Benbouzid MA, Boulaich M, Essakali L, Kzadri M. Solitary bony plasmocytoma of the mandible. Rev Stomatol Chir Maxillofac 2007;108;135-138.

36. Suárez-Roa Mde L, Reveiz L, Ruíz-Godoy Rivera LM, Asbun-Bojalil J, DávilaSerapio JE, Menjívar-Rubio AH, Meneses-García A. Interventions for central giant cell granuloma (CGCG) of the jaws. Cochrane Database Syst Rev 2009;4:CD007404. Review.

37. Ben Slama L, Ruhin B, Zoghbani A. Langerhans cell histiocytosis. Rev Stomatol Chir Maxillofac 2009;110:287-289. Review.

38. Lieberman Ph, Jones CR, Steinman RM, Erlandson RA, Smith J, Gee T. Langerhans Cell (Eosinophilic) Granulomatosis. A Clinicopathologic Study Encompassing 50 Years. Am J Pathol 1996;20:519-525.

39. Scolozzi P, Lombardi T, Monnier P, Jaques B. Multisystem Langerhans' cell histiocytosis (Hand-Schüller-Christian disease) in an adult: a case report and review of the literature. Eur Arch Otorhinolaryngol 2004;261:326-330. Review.

40. Kessler P, Wiltfang J, Schultze-Mosgau S, Neukam FW. Langerhans cell granulomatosis: a case report of polyostotic manifestation in the jaw. Int J Oral Maxillofac Surg 2001;30:359-361. Review.

41. Consolaro A, Sant'Ana E, Lawall MA, Consolaro MF, Bacchi CE. Gingival juvenile xanthogranuloma in an adult patient: case report with immunohistochemical analysis and literature review. Oral Surg Oral Med Oral Pathol Oral Radiol Endod 2009;107:246-252.

42. Driemel O, Braun S, Müller-Richter UD, Behr M, Reichert TE, Kunkel M, Reich R. Historical development of alloplastic temporomandibular joint 
replacement after 1945 and state of the art. Int J Oral Maxillofac Surg 2009;38:909-920. Review.

43. Mortellaro C, Bello L, Lucchina AG, Pucci A. Diagnosis and treatment of familial cherubism characterized by early onset and rapid development. J Craniofac Surg 2009;20:116-120. Review.

44. Feller L, Wood NH, Khammissa RA, Lemmer J, Raubenheimer EJ. The nature of fibrous dysplasia. Head Face Med 2009;5:22-27. Review.

45. Monsour PA, Dalton JB. Chronic recurrent multifocal osteomyelitis involving the mandible: case reports and review of the literature. Dentomaxillofac Radiol 2010;39:184-190. Review.

46. Eyrich GK, Baltensperger MM, Bruder E, Graetz KW. Primary chronic osteomyelitis in childhood and adolescence: a retrospective analysis of 11 cases and review of the literature. J Oral Maxillofac Surg 2003;61:561-573. Review.

47. Musso S, Liolos I, Hofman V, Odin G, Dellamonica P, Hofman P. An oral infection not to be underestimated: actinomycosis. A study of 4 cases with extensive bone necrosis. Ann Pathol 2003;23:161-164.

48. Brook I. Sinusitis of odontogenic origin. Otolaryngol Head Neck Surg 2006;135:349-355. Review.

49. Chakrabarti A, Denning DW, Ferguson BJ, Ponikau J, Buzina W, Kita H, Marple B, Panda N, Vlaminck S, Kauffmann-Lacroix C, Das A, Singh P, TajAldeen SJ, Kantarcioglu AS, Handa KK, Gupta A, Thungabathra M, Shivaprakash MR, Bal A, Fothergill A, Radotra BD. Fungal rhinosinusitis: a categorization and definitional schema addressing current controversies. Laryngoscope 2009;119:1809-1818. Review.

50. Grosjean P, Weber R. Fungus balls of the paranasal sinuses: a review. Eur Arch Otorhinolaryngol 2007;264:461-470. Review.

51. Burnham R, Bridle C. Aspergillosis of the maxillary sinus secondary to a foreign body (amalgam) in the maxillary antrum. Br J Oral Maxillofac Surg 2009; 47:313-315.

52. Mensi M, Salgarello S, Pinsi G, Piccioni M. Mycetoma of the maxillary sinus: endodontic and microbiological correlations. Oral Surg Oral Med Oral Pathol Oral Radiol Endod 2004;98:119-123. 
1099 